\title{
Affluence, Obesity and Non-Communicable Diseases in India
}

\author{
Raghav Gaiha \\ Faculty of Management Studies, \\ University of Delhi, \\ Raghbendra Jha \\ College of Asia \& The Pacific \\ Australian National University, \\ Canberra, \\ \& \\ Vani S. Kulkarni \\ South Asian Studies, \\ Yale University
}

Address for correspondence:

Prof. Raghbendra Jha,

Australia South Asia Research Centre,

Arndt-Corden Division of Economics,

H.C. Coombs Building (09)

Australian National University,

Canberra, ACT 0200, Australia

Phone: + 61261252683

Fax: +61 261250443

Email: r.jha@anu.edu.au 


\title{
Affluence, Obesity and Non-Communicable Diseases in India
}

\begin{abstract}
Recent high rates of economic growth in India have been accompanied by major dietary transitions. Using a nationwide household survey, India Human Development Survey 2005, this paper estimates the impact of such transitions on the incidence of non communicable diseases (NCDs) such as diabetes, coronary heart disease and cancer in India. The estimated deaths from NCDs are projected to rise from 3.78 million in 1990 (40.46 per cent of all deaths) to 7.63 million deaths in 2020 (66.70 per cent of all deaths). The paper also investigates relationships between NCDs and key demographic variables, level of affluence, caste affiliation and geographical concentration of the sample. The paper also models the determinants of these NCDs. The paper concludes with broad policy prescriptions.
\end{abstract}

Keywords: Obesity, affluence, non-communicable diseases, India JEL Classification Code: I12, I19, I32, O15 


\section{Affluence, Obesity and Non-Communicable Diseases in India ${ }^{1}$}

\section{Introduction}

India is currently undergoing a rapid economic and demographic transformation. Since 1980, average living standards have experienced a sustained and rapid rise. The gross domestic product per capita has risen by 230 per cent; a trend rate of 4 per cent annually. Poverty declined at an annual rate of 0.88 per cent from 1983-94, and at a slightly lower rate of 0.77 per cent from 1993-05. Life expectancy has risen from 54 years to 69 years while the (crude) birth rate has fallen from 34 to 22 between 1980-2008. Rapid economic growth has been accompanied by rising urbanization. Between 1980-2000, the share of the urban population rose from 23 to 28 per cent. By 2030, it is likely to be as high as 41 per cent.

The growth momentum was accelerated by wide ranging domestic and external liberalization of the Indian economy in the 1990s. A key feature of the economic transformation has been the change in the nature of the Indian diet. As the global markets integrate and communication becomes better, diet transitions are unavoidable. This results in a move away from inferior to superior foods and a substitution of traditional staples by primary food products that are more prevalent in western diets. These shifts are reflected in higher consumption of proteins, sugars, fats and vegetables.

Some of the underlying factors of this dietary transition include expansion of the middle class, higher female participation, the emergence of nuclear two-income families, a sharp age divide in food preferences (with younger age groups more susceptible to new foods advertised in the media), and a rapid growth of supermarkets and fast-food outlets.

Based on FAOSTAT, some dietary changes observed since the 1980s include:

- The sharp rise in consumption of both animal and vegetable products during the 1980s.

- The consumption of milk as the largest proportional increase among animal products.

- Rice, pulses, wheat, spices, and oils comprising the largest increases among vegetable products.

During the 1990s, significant changes in the pattern of food consumption included:

- A marked increase in the consumption of animal products (especially animal fats) but a relatively modest increase in that of vegetable products.

- Among vegetable products, a large increase in the consumption of wheat, starchy roots, vegetable oils, sugar and sweeteners, and fruits (while that of rice, pulses and other cereals declined).

- Among starchy roots, a sharp increase in potato consumption, given its salience in energy-dense food products (e.g. fries and potato chips).

- A change in the use of wheat due to a move away from the traditional chapatti to more commercialized and westernized bread products.

\footnotetext{
${ }^{1}$ This study was funded by the British Government, under the Foresight Global Food and Farming Futures Project. It has benefited from the advice of L. Haddad, A. Venkatraman and Sonal Desai. Our appreciation of the advice given by Anil Deolalikar cannot be adequately expressed in words. We are also grateful to Ajay Mahal for sharing a recent study. The econometric analysis was carried out competently by Raj Bhatia., with able assistance from Manoj Pandey. We alone are responsible for the views expressed here.
} 
The health implications of the dietary transition are not clear-cut. A more varied and nutritionally balanced diet and higher levels of food hygiene are associated with better health. But there is a trade-off as more energy-dense foods are linked to a higher incidence of dietrelated non-communicable diseases (NCDs) such as diabetes, coronary heart disease and certain types of cancer ${ }^{2}$. Although India lags behind other developing countries in the epidemiological transition - decline in infectious disease mortality compensated increasingly by higher mortality from chronic degenerative NCDs - there is some evidence of the transition taking place. The estimated deaths from NCDs are projected to rise from 3.78 million in 1990 (40.46 per cent of all deaths) to 7.63 million in 2020 (66.70 per cent of all deaths). Worse, many of these deaths occur at relatively early ages; about a quarter of the deaths occurred in the 35-64 age group in urban areas.

In an admirably comprehensive study, Mahal et al. (2009) demonstrate that NCDs constitute a major economic burden in India. They report high levels of out-of-pocket spending by households with members suffering from NCDs, the limited levels of insurance coverage (including subsidised public services) and the income losses that befall affected households. Associated with these costs are risks of catastrophic spending and impoverishment, and of course macroeconomic impacts ${ }^{3}$.

The plan of this paper is as follows. In section II salient features of the household survey on which this analysis is based are described. In section III, prevalence rates of some noncommunicable diseases (e.g. heart disease, diabetes, cancer) and their socio-economic correlates are reviewed. Some of the key interrelationships include demographic aspects of those suffering from such diseases (e.g. gender, age group), their standard of living (or relative affluence), their caste affiliation, location (whether prevalence rates vary between rural and urban areas, as also between urban areas and urban slums), and life-style changes (whether eating out is associated with higher prevalence rates of some of these diseases). In the fourth section, two econometric models are estimated to analyse prevalence of each of these diseases, and the average prevalence for all non-communicable diseases in a household. The final section offers concluding observations from a broad policy perspective.

\section{Data}

Our analysis is based on a nationwide household survey, India Human Development Survey 2005 (IHDS), conducted jointly by University of Maryland and National Council of Applied Economic Research (NCAER).

IHDS covers over 41000 households residing in rural and urban areas, selected from 33 states $^{4}$. The sample comprises 384 districts out of a total of 593 identified in 2001 census. Villages and urban blocks constituted the primary sampling unit from which households were selected.

The rural sample contains about half the households that were interviewed initially by NCAER in 1993-94 in a survey entitled Human Development Profile of India-HDPI-and the

\footnotetext{
2 Non-communicable diseases include conditions such as CVD, cancers, diabetes, chronic obstructive pulmonary disease (COPD), asthma, neuro-psychaitric conditions (e.g. mental disorders), eye conditions, skin diseases, diseases of the digestive system and genitourinary conditions (prostate disorders), among others (Mahal et al. 2009).

${ }^{3}$ For a rigorous and innovative analysis of the overall effect of health on income, including labour productivity and savings, as well as population effects, see Bloom et al. (2009).

${ }^{4}$ This is a summary of the material provided by Sonal Desai.
} 
other half of the sample households was drawn from both districts surveyed in HDPI as well as from districts located in the states and union territories not covered in HDPI. The original HDPI was a random sample of 33,230 households, located in 16 major states, 195 districts and 1765 villages. In states where the 1993-94 survey was conducted and recontact details were available, 13593 households were randomly selected for reinterview in 2005. About 82 per cent of the households were contactable for reinterview resulting in a resurvey of 11,153 original households as well as 2,440 households which had separated from the original households but were still living in the same village.

In each district where reinterviews were conducted, two fresh villages were randomly selected using a probability proportional to size technique. In each village, 20 randomly selected households were selected. Additionally, 3,993 households were randomly selected from the states where the 1993-94 survey was not conducted, or where recontact information was not available.

In order to draw a random sample of urban households, all urban areas in a state were listed in the order of their size with number of blocks drawn from each urban area allocated based on probability proportional to size. After determining the number of blocks, the enumeration blocks were selected randomly. From these enumeration blocks (of about 150-200 households), a complete household listing was obtained and a sample of 15 households was selected per block.

Comparison of IHDS data with the National Sample Survey or NSS (2004-05), National Family Health Survey III (2005-06) and Census (2001) confirms the robustness of IHDS data. For example, IHDS sample distribution on urban residence, caste and religion is remarkably similar to NSS and NFHS-III, although all three surveys (IHDS, NSS and NFHS) have higher proportions of households claiming Scheduled Caste status than enumerated in Census (2001).

As incidence of NCDs is self-reported, it has familiar limitations. Some elaboration may be helpful. The main problem is that we only observe the response to the statement: "I have disease $\mathrm{Z}$ '. If $\mathrm{X}=1$ when the response is "yes", then we can decompose the probability into $\mathrm{P}(\mathrm{X})=\mathrm{P}(\mathrm{X} \mid \mathrm{Z}) * \mathrm{P}(\mathrm{Z})+\mathrm{P}(\mathrm{X} \mid \sim \mathrm{Z}) * \mathrm{P}(\sim \mathrm{Z})$. We are interested in measuring $\mathrm{P}(\mathrm{Z})$ but only observe $\mathrm{P}(\mathrm{X})$ having to contend with false negatives $\mathrm{P}(\mathrm{X} \mid \mathrm{Z}) \neq 1$ generally from ignorance and misdiagnoses or false positives $\mathrm{P}(\mathrm{X} \mid \sim \mathrm{Z}) \neq 0$ from either mis-diagnosis or hypochondria (Chaudhury et al. 2008).

\section{Correlates of NCDs}

As the classification into different NCDs (high blood pressure, heart disease and diabetes) is not mutually exclusive, let us first consider cases that overlap. As noted earlier, the analysis is confined to those $>22$ years. In Table 1, we focus on the overlap between high blood pressure and heart disease, and in Table 2 between heart disease and diabetes ${ }^{5}$. As cancer does not overlap much with either heart disease or diabetes, it is not considered here. There is, however, some overlap with high blood pressure (about 10 per cent of cancer patients also suffered from high blood pressure).

\footnotetext{
${ }^{5}$ These overlaps point to common underlying factors. For example, the underlying causes of diabetes overlap with those of cardiovascular disease (Bajpai et al. 2010).
} 
As Table 1 shows, the overlap between high blood pressure and heart disease was about 21 per cent. The overlap between high blood pressure and diabetes was even higher (about 33 per cent). However, the overlap between heart disease and diabetes was somewhat low (about 7 per cent). Whatever the plausibility of these estimates, the importance of competing medical risks in classifying individuals as well as in designing health policies is reinforced (Mahal et al. 2009).

Table 1

Overlap between High Blood Pressure and Heart Disease

\begin{tabular}{|c|c|c|c|c|}
\hline \multirow{2}{*}{$\begin{array}{l}\text { Whether Suffering from } \\
\text { High Blood Pressure? }\end{array}$} & \multicolumn{3}{|c|}{ Whether Suffering from Heart Disease? } & \multirow{2}{*}{ Total } \\
\cline { 2 - 4 } & No & Yes & Cured & \\
\hline \multirow{2}{*}{ No } & 90.98 & 8.46 & 0.57 & 100 \\
& $(78.25)$ & $(78.86)$ & $(91.21)$ & $(78.37)$ \\
\hline \multirow{2}{*}{ Yes } & 91.51 & 8.29 & 0.20 & 100 \\
& $(21.33)$ & $(\mathbf{2 0 . 9 6 )}$ & $(8.79)$ & $(21.24)$ \\
\hline \multirow{2}{*}{ Cured } & 96.21 & 3.79 & 0.0 & 100 \\
& $(0.42)$ & $(0.18)$ & $(0.0)$ & $(0.39)$ \\
\hline \multirow{2}{*}{ Total } & 91.11 & 8.40 & 0.49 & 100 \\
& $(100)$ & $(100)$ & $(100)$ & $(100)$ \\
\hline
\end{tabular}

Table 2

Overlap between High Blood Pressure and Diabetes

\begin{tabular}{|c|c|c|c|c|}
\hline \multirow{2}{*}{$\begin{array}{l}\text { Whether Suffering from } \\
\text { High Blood Pressure? }\end{array}$} & \multicolumn{3}{|c|}{ Whether Suffering from Diabetes? } & \multirow{2}{*}{ Total } \\
\hline & No & Yes & Cured & \\
\hline No & $\begin{array}{l}88.74 \\
(79.77)\end{array}$ & $\begin{array}{c}10.96 \\
(66.74)\end{array}$ & $\begin{array}{c}0.29 \\
(89.96)\end{array}$ & $\begin{array}{c}100 \\
(78.12)\end{array}$ \\
\hline Yes & $\begin{array}{c}80.11 \\
(19.80)\end{array}$ & $\begin{array}{c}19.80 \\
(33.14)\end{array}$ & $\begin{array}{c}0.10 \\
(8.07)\end{array}$ & $\begin{array}{c}100 \\
(21.49)\end{array}$ \\
\hline Cured & $\begin{array}{l}94.93 \\
(0.43)\end{array}$ & $\begin{array}{c}3.79 \\
(0.12)\end{array}$ & $\begin{array}{c}1.28 \\
(1.97)\end{array}$ & $\begin{array}{c}100 \\
(0.39)\end{array}$ \\
\hline Total & $\begin{array}{l}86.91 \\
(100)\end{array}$ & $\begin{array}{l}12.83 \\
(100)\end{array}$ & $\begin{array}{l}0.26 \\
(100)\end{array}$ & $\begin{array}{l}100 \\
(100)\end{array}$ \\
\hline
\end{tabular}

Table 3

Overlap between Heart Disease and Diabetes

\begin{tabular}{|c|c|c|c|c|}
\hline \multirow{2}{*}{$\begin{array}{l}\text { Whether Suffering from } \\
\text { Heart Disease? }\end{array}$} & \multicolumn{3}{|c|}{ Whether Suffering from Diabetes? } & \multirow{2}{*}{ Total } \\
\cline { 2 - 5 } & No & Yes & Cured & 100 \\
\hline \multirow{2}{*}{ No } & $\begin{array}{c}86.94 \\
(90.92)\end{array}$ & $\begin{array}{c}12.80 \\
(93.01)\end{array}$ & 0.26 \\
& 89.28 & 10.49 & $0.49)$ & $1.18)$ \\
\hline \multirow{2}{*}{ Yes } & $(8.48)$ & $(6.93)$ & $(7.54)$ & $(8.29)$ \\
\hline \multirow{2}{*}{ Cured } & 97.52 & 1.52 & 0.96 & 100 \\
& $(0.59)$ & $(0.06)$ & $(1.97)$ & $(0.53)$ \\
\hline Total & 87.19 & 12.55 & 0.26 & 100 \\
& $(100)$ & $(100)$ & $(100)$ & $(100)$ \\
\hline
\end{tabular}




\section{(a) Demographic Aspects}

Our review is confined to whether prevalence rates of different NCDs vary by gender and age group. Note that the analysis is confined to household members $>22$ years.

Table 4

Prevalence of High Blood Pressure (HBP) by Gender

\begin{tabular}{|c|c|c|c|}
\hline $\begin{array}{c}\text { Whether Suffering from } \\
\text { HBP? }\end{array}$ & Male & Female & Total \\
\hline \multirow{2}{*}{ No } & 47.13 & 52.87 & 100 \\
& $(76.08)$ & $(70.34)$ & $(72.93)$ \\
\hline \multirow{2}{*}{ Yes } & 39.76 & 60.24 & 100 \\
& $(23.42)$ & $(29.25)$ & $(26.61)$ \\
\hline \multirow{2}{*}{ Cured } & 49.91 & 50.09 & 100 \\
& $(0.50)$ & $(0.42)$ & $(0.46)$ \\
\hline \multirow{2}{*}{ Total } & 45.18 & 54.82 & 100 \\
& $(100)$ & $(100)$ & $(100)$ \\
\hline
\end{tabular}

Table 5

Prevalence of HBP by Age Group

\begin{tabular}{|c|c|c|c|}
\hline $\begin{array}{c}\text { Whether Suffering from } \\
\text { HBP? }\end{array}$ & \multicolumn{2}{|c|}{ Age Group (years) } & \\
\hline \multirow{2}{*}{ No } & 43.63 & 545 & 100 \\
& $(81.86)$ & $(67.26)$ & $(72.93)$ \\
\hline \multirow{2}{*}{ Yes } & 25.62 & 74.38 & 100 \\
& $(17.54)$ & $(32.38)$ & $(26.61)$ \\
\hline \multirow{2}{*}{ Cured } & 50.93 & 49.07 & 100 \\
& $(0.60)$ & $(0.37)$ & $(0.46)$ \\
\hline \multirow{2}{*}{ Total } & 38.87 & 61.13 & 100 \\
& $(100)$ & $(100)$ & $(100)$ \\
\hline
\end{tabular}

Table 6

Prevalence of Heart Disease by Gender

\begin{tabular}{|c|c|c|c|}
\hline $\begin{array}{c}\text { Whether Suffering from } \\
\text { Heart Disease? }\end{array}$ & Male & Female & Total \\
\hline \multirow{2}{*}{ No } & 45.70 & 54.30 & 100 \\
& $(90.01)$ & $(90.26)$ & $(90.14)$ \\
\hline \multirow{2}{*}{ Yes } & 44.82 & 55.18 & 100 \\
& $(9.01)$ & $(9.36)$ & $(9.20)$ \\
\hline \multirow{2}{*}{ Cured } & 68.50 & 31.50 & 100 \\
& $(0.98)$ & $(0.38)$ & $(0.65)$ \\
\hline \multirow{2}{*}{ Total } & 45.77 & 54.23 & 100 \\
& $(100)$ & $(100)$ & $(100)$ \\
\hline
\end{tabular}

About 26 per cent of the sample individuals suffered from high blood pressure. Of these a large majority (over 60 per cent) were females. As shown in Table 5, a large majority (over 74 per cent) were also in the older age-group $>45$ years. 
Table 7

Prevalence of Heart Disease by Age Group (Years)

\begin{tabular}{|c|c|c|c|}
\hline $\begin{array}{c}\text { Whether Suffering from } \\
\text { Heart Disease? }\end{array}$ & $23-45$ & $>45$ & \\
\hline \multirow{2}{*}{ No } & 39.88 & 60.12 & 100 \\
& $(91.26)$ & $(89.42)$ & $(90.14)$ \\
\hline \multirow{2}{*}{ Yes } & 33.89 & 66.11 & 100 \\
& $(7.92)$ & $(10.04)$ & $(9.20)$ \\
\hline \multirow{2}{*}{ Cured } & 49.77 & 50.23 & 100 \\
& $(0.82)$ & $(0.54)$ & $(0.65)$ \\
\hline \multirow{2}{*}{ Total } & 39.40 & 60.60 & 100 \\
& $(100)$ & $(100)$ & $(100)$ \\
\hline
\end{tabular}

The prevalence rate of heart disease (over 9 per cent) was considerably lower relative to high blood pressure. Of these the majority (over 55 per cent) were females. As in the case of high blood pressure, the majority of those suffering from heart disease were in the older age-group $>45$ years.

We note from Table 8 that the prevalence of diabetes was just under 16 per cent of the sample. Of those suffering from diabetes, the majority (about 56 per cent) were males. Table 9 gives the age distribution. As in the case of high blood pressure and heart disease, the majority of diabetics were in the older age-group $>45$ years.

As shown in Table 10, the prevalence of cancer (about 1.25 per cent) is relatively low. The majority of those reported to be suffering from it (about 58 per cent) were males. Table 11 shows that the majority were also in the older age-group $>45$ years.

\section{(b) Affluence and NCDs}

As some of the NCDs (e.g. heart disease) are closely associated with prosperity, life-style changes and dietary transition, we examine below the links between these diseases and indicators of living standards ${ }^{6}$. A diagrammatic representation of risk of diet-related factors associated with selected NCDs is given in Figures 1 to 3 .

Often measurement of living standards in a money metric is problematic (e.g. because of the difficulties in measuring inputs and outputs that are not marketed). To overcome this difficulty, some proximate indicators (e.g. caste and location) are also considered

Using the official poverty line, we have classified individuals by their poverty status ${ }^{7}$. Table 12 shows that a vast majority of those reported to be suffering from high blood pressure (over 90 per cent) were non-poor. Also, among the non-poor, well over a quarter suffered from high blood pressure, while among the poor just one-fifth did.

\footnotetext{
${ }^{6}$ It is widely believed that populations from South, East and Southeast Asia have a heightened susceptibility to diabetes when combined with unhealthy diets, low levels of physical activity, and smoking (Bajpai et al. 2010).

${ }^{7}$ The average poverty line is Rs 356 per person per month in rural areas (at 2004-05 prices), rs 538 in urban areas. State-wise poverty lines range from Rs 292 to Rs 478 in rural areas, and Rs 378 to Rs 668 in urban areas (Desai et al. 2010).
} 


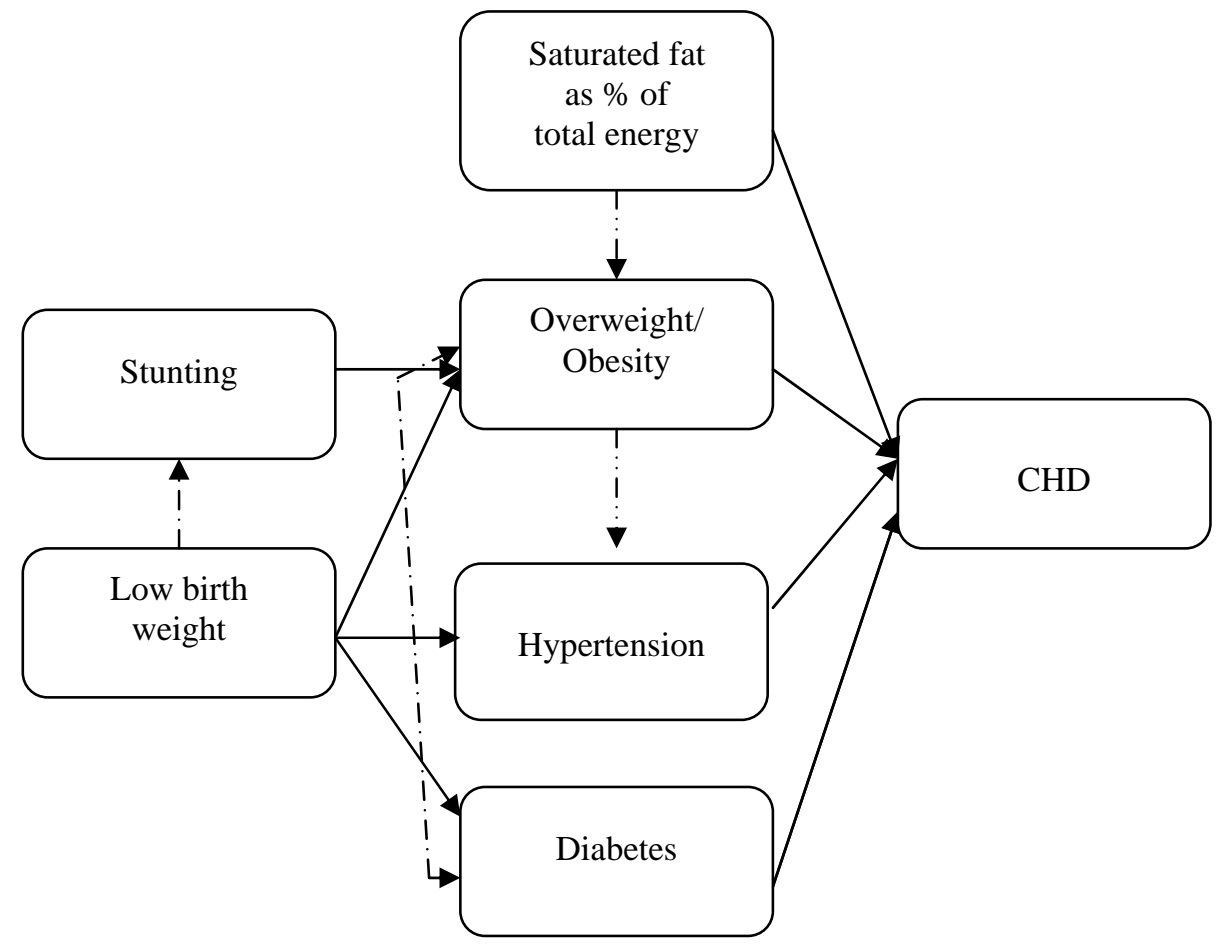

Figure 1: Relative risks for coronary heart disease. $\mathrm{CHD}=$ coronary heart disease

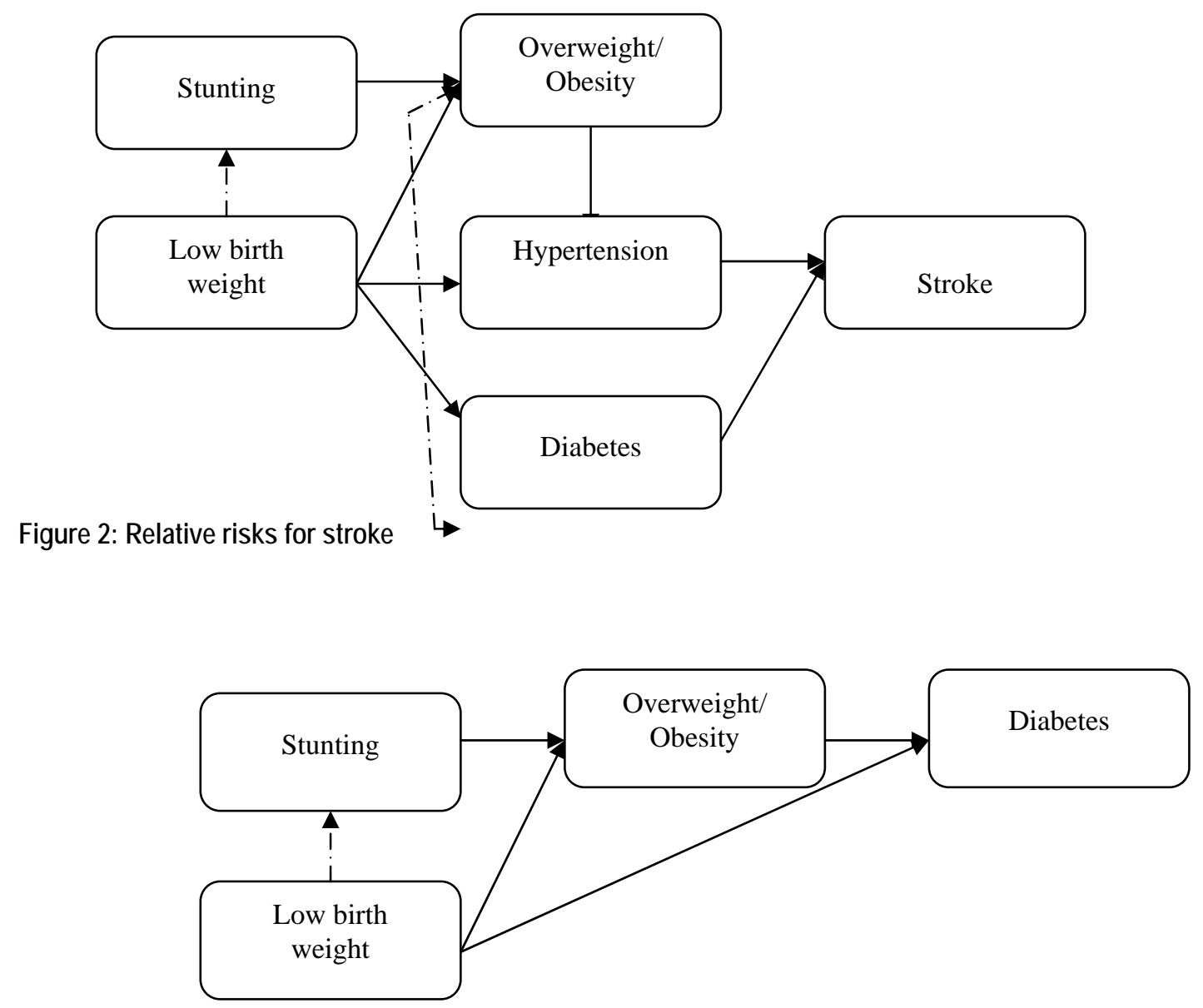

Figure 3: Relative risks for diabetes

Source: Adapted from Popkin et al. (2001) 
Table 8

Prevalence of Diabetes by Gender

\begin{tabular}{|c|c|c|c|}
\hline $\begin{array}{c}\text { Whether Suffering from } \\
\text { Diabetes? }\end{array}$ & Male & Fender & Total \\
\hline No & $\begin{array}{c}43.74 \\
(80.40)\end{array}$ & $\begin{array}{c}56.26 \\
(87.05)\end{array}$ & $\begin{array}{c}100 \\
(84.01)\end{array}$ \\
\hline Yes & 55.89 & 44.11 & 100 \\
& $(19.14)$ & $(12.72)$ & $(15.65)$ \\
\hline \multirow{2}{*}{ Cured } & 62.74 & 37.26 & 100 \\
& $(0.46)$ & $(0.23)$ & $(0.34)$ \\
\hline \multirow{2}{*}{ Total } & 45.71 & 54.29 & 100 \\
& $(100)$ & $(100)$ & $(100)$ \\
\hline
\end{tabular}

Table 9

Prevalence of Diabetes by Age Group (Years)

\begin{tabular}{|c|c|c|c|}
\hline $\begin{array}{c}\text { Whether Suffering from } \\
\text { Diabetes? }\end{array}$ & $23-45$ & $>45$ & Total \\
\hline \multirow{2}{*}{ No } & $\begin{array}{c}42.40 \\
(90.96)\end{array}$ & $\begin{array}{c}57.60 \\
(79.54)\end{array}$ & 100 \\
& 22.23 & 77.77 & $(84.01)$ \\
\hline \multirow{2}{*}{ Yes } & $(8.89)$ & $(20.01)$ & 100 \\
& 18.05 & 81.95 & $(15.65)$ \\
\hline Cured & $(0.15)$ & $(0.45)$ & 100 \\
& 39.16 & 60.84 & $(0.34)$ \\
\hline \multirow{2}{*}{ Total } & $(100)$ & $(100)$ & 100 \\
& & & $(100)$ \\
\hline
\end{tabular}

As the poverty line allows for a bare subsistence living, a more detailed expenditure classification is employed in Table $13^{8}$. The contrast is once again striking. About 85 per cent of those suffering from high blood pressure were in the upper tail of the expenditure distribution (>Rs 500 per capita per month) while just over 2 per cent were in the lower tail ( $<$ Rs 300 per capita per month). What is also striking is that as the expenditure interval rises the proportions suffering from high blood pressure also rises, with over 36 per cent of those in the highest expenditure interval reporting this condition- more than three times larger than among those in the lower tail.

Table 10

Prevalence of Cancer by Gender

\begin{tabular}{|c|c|c|c|}
\hline $\begin{array}{c}\text { Whether Suffering from } \\
\text { Cancer? }\end{array}$ & \multicolumn{2}{|c|}{ Mender } & Total \\
\hline \multirow{2}{*}{ No } & 45.48 & 54.52 & 100 \\
& $(98.34)$ & $(98.86)$ & $(98.62)$ \\
\hline \multirow{2}{*}{ Yes } & 57.86 & 42.14 & 100 \\
& $(1.57)$ & $(0.96)$ & $(1.24)$ \\
\hline \multirow{2}{*}{ Cured } & 29.61 & 70.39 & 100 \\
& $(0.09)$ & $(0.19)$ & $(0.14)$ \\
\hline \multirow{2}{*}{ Total } & 45.61 & 54.39 & 100 \\
& $(100)$ & $(100)$ & $(100)$ \\
\hline
\end{tabular}

\footnotetext{
${ }^{8}$ Note that per capita expenditure is preferred to per capita income as a welfare indicator.
} 
Table 11

Prevalence of Cancer by Age Group (Years)

\begin{tabular}{|c|c|c|c|}
\hline \multirow{2}{*}{$\begin{array}{c}\text { Whether Suffering } \\
\text { from Cancer? }\end{array}$} & \multicolumn{2}{|c|}{ Age Group } & \multirow{2}{*}{ Total } \\
\cline { 2 - 3 } & $23-45$ & $>45$ & 100 \\
\hline \multirow{2}{*}{ No } & 39.50 & 60.50 & $(98.62)$ \\
\hline \multirow{2}{*}{ Yes } & $(98.82)$ & $(98.49)$ & 100 \\
& 30.93 & 69.02 & $(1.24)$ \\
\hline \multirow{2}{*}{ Cured } & $(0.97)$ & $(1.41)$ & 100 \\
& 57.95 & 42.05 & $(0.14)$ \\
\hline \multirow{2}{*}{ Total } & $(0.21)$ & $(0.10)$ & 100 \\
& 39.42 & 60.58 & $(100)$ \\
\hline
\end{tabular}

Table 12

Prevalence of HBP by Poverty Status

\begin{tabular}{|c|c|c|c|}
\hline \multirow{2}{*}{$\begin{array}{c}\text { Whether Suffering from } \\
\text { HBP? }\end{array}$} & \multicolumn{2}{|c|}{ Below Poverty Line } & \multirow{2}{*}{ Total } \\
\cline { 2 - 4 } & No & Yes & 100 \\
No & $\begin{array}{c}84.69 \\
(71.61)\end{array}$ & $\begin{array}{c}15.31 \\
(81.21)\end{array}$ & $(72.93)$ \\
\hline Yes & 90.41 & 9.59 & 100 \\
& $(27.89)$ & $(18.56)$ & $(26.61)$ \\
\hline \multirow{2}{*}{ Cured } & 93.02 & 6.98 & 100 \\
& $(0.49)$ & $(0.23)$ & $(0.46)$ \\
\hline \multirow{2}{*}{ Total } & 86.25 & 13.75 & 100 \\
& $(100)$ & $(100)$ & $(100)$ \\
\hline
\end{tabular}

Table 13

Prevalence of HBP by Monthly Per Capita Expenditure (Rs)

\begin{tabular}{|c|c|c|c|c|c|}
\hline \multirow{2}{*}{$\begin{array}{c}\text { Whether } \\
\text { Suffering from } \\
\text { HBP? }\end{array}$} & \multicolumn{4}{|c|}{ Monthly Per Capita Expenditure (Rs) } & \multirow{2}{*}{ Total } \\
\hline & $<300$ & $300-500$ & $500-1000$ & $>1000$ & \\
\hline No & $\begin{array}{c}6.65 \\
(88.90)\end{array}$ & $\begin{array}{c}21.17 \\
(81.97)\end{array}$ & $\begin{array}{c}41.91 \\
(74.93)\end{array}$ & $\begin{array}{c}30.27 \\
(63.23)\end{array}$ & $\begin{array}{c}100 \\
(72.93)\end{array}$ \\
\hline Yes & $\begin{array}{c}2.25 \\
(10.98)\end{array}$ & $\begin{array}{c}12.61 \\
(17.81)\end{array}$ & $\begin{array}{c}37.63 \\
(24.54)\end{array}$ & $\begin{array}{c}47.52 \\
(36.22)\end{array}$ & $\begin{array}{c}100 \\
(26.61)\end{array}$ \\
\hline Cured & $\begin{array}{c}1.46 \\
(0.12)\end{array}$ & $\begin{array}{c}9.17 \\
(0.22)\end{array}$ & $\begin{array}{l}46.71 \\
(0.52)\end{array}$ & $\begin{array}{l}42.66 \\
(0.56)\end{array}$ & $\begin{array}{c}100 \\
(0.46)\end{array}$ \\
\hline Total & $\begin{array}{l}5.45 \\
(100)\end{array}$ & $\begin{array}{l}18.83 \\
(100)\end{array}$ & $\begin{array}{l}40.79 \\
(100)\end{array}$ & $\begin{array}{l}34.92 \\
(100)\end{array}$ & $\begin{array}{c}100 \\
(100)\end{array}$ \\
\hline
\end{tabular}

Another correlate of the standard of living - although aggregate in nature - is location. In Table 14, individuals are classified as living in rural, urban and urban slums, with those living in rural and urban slums as generally worse-off than those living in urban areas (Desai et al. 2010). The pattern is striking, with the largest proportion of those suffering from high blood pressure (about 39 per cent) in urban areas and then those in urban slums (well over 35 per cent). So relative affluence and life styles appear to be closely linked to the prevalence of high blood pressure. 
Table 14

Prevalence of HBP by Location

\begin{tabular}{|c|c|c|c|c|}
\hline \multirow{2}{*}{$\begin{array}{c}\text { Whether Suffering } \\
\text { from HBP? }\end{array}$} & \multicolumn{3}{|c|}{ Location } & \multirow{2}{*}{ Total } \\
\cline { 2 - 4 } & Rural & Urban & Urban Slum & \\
\hline \multirow{2}{*}{ No } & 74.75 & 24.04 & 1.20 & 100 \\
& $(78.26)$ & $(60.56)$ & $(63.77)$ & $(72.93)$ \\
\hline \multirow{2}{*}{ Yes } & 21.41 & 38.70 & 35.55 & 26.61 \\
& $(14.91)$ & $(11.21)$ & $(0.49)$ & $(26.61)$ \\
\hline \multirow{2}{*}{ Cured } & 50.95 & 46.98 & 2.06 & 100 \\
& $(0.33)$ & $(0.74)$ & $(0.68)$ & $(0.46)$ \\
\hline \multirow{2}{*}{ Total } & 69.67 & 28.96 & 1.38 & 100 \\
& $(100)$ & $(100)$ & $(100)$ & $(100)$ \\
\hline
\end{tabular}

Yet another correlate of the standard of living is caste. Following a common classification in Table 15, individuals are classified into Scheduled Castes (SCs), Scheduled Tribes (STs), Other Backward Castes (OBCs) and Others. The STs are typically the most poverty prone, followed by the SCs, and then the OBCs (Desai et al. 2010).

Table 15

Prevalence of HBP by Caste

\begin{tabular}{|c|c|c|c|c|c|}
\hline $\begin{array}{l}\text { Whether Suffering } \\
\text { from HBP? }\end{array}$ & SCs & STs & OBCs & Others & Total \\
\hline No & $\begin{array}{c}20.67 \\
(78.99)\end{array}$ & $\begin{array}{c}5.06 \\
(89.15) \\
\end{array}$ & $\begin{array}{c}38.43 \\
(75.04) \\
\end{array}$ & $\begin{array}{c}35.84 \\
(66.30) \\
\end{array}$ & $\begin{array}{c}100 \\
(72.93)\end{array}$ \\
\hline Yes & $\begin{array}{c}14.76 \\
(20.59)\end{array}$ & $\begin{array}{c}1.63 \\
(10.49)\end{array}$ & $\begin{array}{c}34.23 \\
(24.39)\end{array}$ & $\begin{array}{c}49.37 \\
(33.32)\end{array}$ & $\begin{array}{c}100 \\
(26.61)\end{array}$ \\
\hline Cured & $\begin{array}{l}17.50 \\
(0.42)\end{array}$ & $\begin{array}{l}3.26 \\
(0.36)\end{array}$ & $\begin{array}{l}46.97 \\
(0.57)\end{array}$ & $\begin{array}{l}32.27 \\
(0.37)\end{array}$ & $\begin{array}{c}100 \\
(0.46)\end{array}$ \\
\hline Total & $\begin{array}{l}19.08 \\
(100)\end{array}$ & $\begin{array}{l}4.14 \\
(100)\end{array}$ & $\begin{array}{l}37.35 \\
(100)\end{array}$ & $\begin{array}{l}39.42 \\
(100)\end{array}$ & $\begin{array}{c}100 \\
(100)\end{array}$ \\
\hline
\end{tabular}

The pattern is suggestive as the largest proportion of those suffering from high blood pressure were among Others, followed by OBCs and then the SCs and STs. If Others are least prone to poverty and generally the most affluent, there is further support for a strong link between (relative) affluence and this condition. This is further reinforced by the fact that nearly one third of Others reported to be affected by this condition.

Table 16

Prevalence of Heart Disease by Poverty Status

\begin{tabular}{|c|c|c|c|}
\hline \multirow{2}{*}{$\begin{array}{c}\text { Whether Suffering } \\
\text { from Heart Disease? }\end{array}$} & \multicolumn{2}{|c|}{ Below Poverty Line } & \multirow{2}{*}{ Total } \\
\cline { 2 - 3 } & No & Yes & 100 \\
\hline \multirow{2}{*}{ No } & 85.46 & 14.54 & $(90.14)$ \\
\hline \multirow{2}{*}{ Yes } & $(89.60)$ & $(93.51)$ & 100 \\
& 90.55 & 9.45 & $(9.20)$ \\
\hline \multirow{2}{*}{ Cured } & $(9.69)$ & $(6.20)$ & 100 \\
& 93.81 & 6.19 & $(0.65)$ \\
\hline \multirow{2}{*}{ Total } & $(0.71)$ & $(0.29)$ & 100 \\
& 85.99 & 14.01 & $(100)$ \\
\hline
\end{tabular}


As in the case of high blood pressure, a vast majority of those reported to be suffering from heart disease (over 90 per cent ) were non-poor.

Using the more detailed expenditure classification in Table 17, we find that the majority of those suffering from heart disease (about 49 per cent) were in the upper tail of the income distribution while barely 2 per cent were in the lower tail. Also, the proportion suffering from this condition rises with the expenditure interval.

Table 17

Prevalence of Heart Disease by Monthly Per Capita Expenditure (Rs)

\begin{tabular}{|c|c|c|c|c|c|}
\hline \multirow{2}{*}{$\begin{array}{c}\text { Whether } \\
\text { Suffering from } \\
\text { Heart Disease? }\end{array}$} & \multicolumn{4}{|c|}{ Monthly Per Capita Expenditure (Rs) } & \multirow{2}{*}{ Total } \\
\cline { 2 - 5 } & $<300$ & $300-500$ & $500-1000$ & $>1000$ & \\
\hline No & $\begin{array}{c}6.01 \\
(96.63)\end{array}$ & $\begin{array}{c}19.80 \\
(93.82)\end{array}$ & $\begin{array}{c}41.30 \\
(90.80)\end{array}$ & $\begin{array}{c}32.89 \\
(86.27)\end{array}$ & $\begin{array}{c}100 \\
(90.14)\end{array}$ \\
\hline \multirow{2}{*}{ Yes } & 1.99 & $\begin{array}{c}12.29 \\
(5.95)\end{array}$ & $\begin{array}{c}36.91 \\
(8.29)\end{array}$ & $\begin{array}{c}48.81 \\
(13.07)\end{array}$ & $\begin{array}{c}100 \\
(9.20)\end{array}$ \\
\hline \multirow{2}{*}{ Cured } & $\begin{array}{c}(3.26) \\
(0.11)\end{array}$ & $\begin{array}{c}6.82 \\
(0.23)\end{array}$ & $\begin{array}{c}57.82 \\
(0.92)\end{array}$ & $\begin{array}{c}34.46 \\
(0.65)\end{array}$ & $\begin{array}{c}100 \\
(0.65)\end{array}$ \\
\hline \multirow{2}{*}{ Total } & 5.61 & 19.03 & 41.00 & 34.36 & 100 \\
\hline
\end{tabular}

Table 18 points to the largest concentration of those suffering from heart disease in rural areas, followed by urban areas. However, the shares of those living in different locations with this condition vary over a small range, with the largest proportion in urban slums.

Table 18

Prevalence of Heart Disease by Location

\begin{tabular}{|c|c|c|c|c|}
\hline \multirow{2}{*}{$\begin{array}{c}\text { Whether Suffering } \\
\text { from Heart } \\
\text { Disease? }\end{array}$} & \multicolumn{3}{|c|}{ Location } & \multirow{2}{*}{ Total } \\
\cline { 2 - 4 } & Rural & Urban & Urban Slums & \\
\hline \multirow{2}{*}{ No } & $\begin{array}{c}70.95 \\
(91.78)\end{array}$ & $\begin{array}{c}27.75 \\
(86.40)\end{array}$ & $\begin{array}{c}1.29 \\
(85.90)\end{array}$ & 100 \\
& 57.21 & 41.02 & 1.78 & $(90.14)$ \\
\hline \multirow{2}{*}{ Yes } & $(7.56)$ & $(13.04)$ & $(12.06)$ & 100 \\
& 70.71 & 25.06 & 4.23 & $(9.20)$ \\
\hline \multirow{2}{*}{ Cured } & $(0.66)$ & $(0.56)$ & $(2.04)$ & 100 \\
& 69.69 & 28.96 & 1.35 & $10.65)$ \\
\hline \multirow{2}{*}{ Total } & $(100)$ & $(100)$ & $(100)$ & $(100)$ \\
\hline
\end{tabular}

The cross-classification of those suffering from heart disease by caste in Table 19 points to the (relatively) most affluent group of Others accounting for the majority of those affected by this condition (over 52 per cent) while the most disadvantaged (the STs) accounted for a little under 1.50 per cent. The proportion of Others who reported this disease was also the highest (but only slightly higher than the corresponding shares of SCs and OBCs). 
Table 19

Prevalence of Heart Disease by Caste

\begin{tabular}{|c|c|c|c|c|c|}
\hline $\begin{array}{c}\text { Whether Suffering } \\
\text { from Heart Disease? }\end{array}$ & SCs & STs & OBCs & Others & Total \\
\hline \multirow{2}{*}{ No } & $\begin{array}{c}19.83 \\
(92.27)\end{array}$ & $\begin{array}{c}4.36 \\
(92.34)\end{array}$ & $\begin{array}{c}38.27 \\
(91.89)\end{array}$ & $\begin{array}{c}37.54 \\
(87.16)\end{array}$ & $\begin{array}{c}100 \\
(90.14)\end{array}$ \\
\hline \multirow{2}{*}{ Yes } & 15.27 & 1.36 & 30.99 & 52.38 & 100 \\
& $(7.26)$ & $(2.93)$ & $(7.60)$ & $(12.42)$ & $(9.20)$ \\
\hline \multirow{2}{*}{ Cured } & 14.17 & 30.87 & 29.66 & 25.30 & 100 \\
& $(0.48)$ & $(4.73)$ & $(0.52)$ & $(0.43)$ & $(0.65)$ \\
\hline \multirow{2}{*}{ Total } & 19.37 & 4.26 & 37.55 & 38.82 & 100 \\
& $(100)$ & $(100)$ & $(100)$ & $(100)$ & $(100)$ \\
\hline
\end{tabular}

As in other NCDs, a vast majority of diabetics (about 93 per cent) were non-poor. Also, the proportion of the non-poor reporting this condition was twice as high as among the poor. In the more detailed expenditure classification in Table 21, over 48 per cent of the diabetics were in the highest expenditure interval (>Rs 1000 per capita per month) while just under 2 per cent were in the lowest interval ( $<$ Rs 300 per capita per month). Also, the proportion of those reporting this condition in the highest expenditure interval was nearly 5 times that in the lowest interval.

Table 20

Prevalence of Diabetes by Poverty Status

\begin{tabular}{|c|c|c|c|}
\hline \multirow{2}{*}{$\begin{array}{c}\text { Whether Suffering from } \\
\text { Diabetes? }\end{array}$} & \multicolumn{2}{|c|}{ Below Poverty Line } & \multirow{2}{*}{ Total } \\
\cline { 2 - 4 } & No & Yes & 100 \\
No & 84.69 & 15.31 & $(84.01)$ \\
\hline \multirow{2}{*}{ Yes } & $(82.77)$ & $(91.63)$ & 100 \\
& 92.73 & 7.27 & $(15.65)$ \\
\hline \multirow{2}{*}{ Cured } & $(16.89)$ & $(8.10)$ & 100 \\
& 88.72 & 11.28 & $(0.34)$ \\
\hline \multirow{2}{*}{ Total } & $(0.35)$ & $(0.27)$ & 100 \\
& 85.96 & 14.04 & $(100)$ \\
\hline
\end{tabular}

Table 21

Prevalence of Diabetes by Per Capita Monthly Expenditure (Rs)

\begin{tabular}{|c|c|c|c|c|c|}
\hline \multirow{2}{*}{$\begin{array}{c}\text { Whether Suffering } \\
\text { from Diabetes? }\end{array}$} & \multicolumn{4}{|c|}{ Monthly Per Capita Expenditure (Rs) } & \multirow{2}{*}{ Total } \\
\cline { 2 - 5 } & $<300$ & $300-500$ & $500-1000$ & $>1000$ & \\
\hline \multirow{2}{*}{ No } & 6.36 & 20.55 & 41.25 & 31.84 & 100 \\
& $(95.17)$ & $(90.65)$ & $(84.80)$ & $(77.59)$ & $(84.01)$ \\
\hline \multirow{2}{*}{ Yes } & 1.62 & 11.11 & 39.04 & 48.23 & 100 \\
& $(4.51)$ & $(9.13)$ & $(14.95)$ & $(21.90)$ & $(15.65)$ \\
\hline \multirow{2}{*}{ Cured } & 5.31 & 12.36 & 29.94 & 52.39 & 100 \\
& $(0.32)$ & $(0.22)$ & $(0.25)$ & $(0.51)$ & $(0.34)$ \\
\hline \multirow{2}{*}{ Total } & 5.61 & 19.05 & 40.87 & 34.47 & 100 \\
& $(100)$ & $(100)$ & $(100)$ & $(100)$ & $(100)$ \\
\hline
\end{tabular}


Table 22

Prevalence of Diabetes by Location

\begin{tabular}{|c|c|c|c|c|}
\hline \multirow{2}{*}{$\begin{array}{c}\text { Whether Suffering } \\
\text { from Diabetes? }\end{array}$} & \multicolumn{3}{|c|}{ Location } & \multirow{2}{*}{ Total } \\
\cline { 2 - 4 } & Rural & Urban & Urban Slums & \\
\hline \multirow{3}{*}{ No } & 72.36 & 26.24 & 1.41 & 100 \\
& $(87.48)$ & $(75.54)$ & $(88.40)$ & $(84.01)$ \\
\hline \multirow{2}{*}{ Yes } & 54.35 & 44.66 & 0.99 & 100 \\
& $(12.24)$ & $(23.96)$ & $(11.60)$ & $(15.65)$ \\
\hline \multirow{2}{*}{ Cured } & 56.26 & 43.74 & 0.0 & 100 \\
& $(0.27)$ & $(0.50)$ & $(0.0)$ & $(0.34)$ \\
\hline \multirow{2}{*}{ Total } & 69.48 & 29.18 & 1.34 & 100 \\
& $(100)$ & $(100)$ & $(100)$ & $(100)$ \\
\hline
\end{tabular}

Diabetes is widely prevalent in rural areas (over 54 per cent), followed closely by urban areas (about 45 per cent) ${ }^{9}$. However, among those in urban areas, about a quarter reported this condition, more than twice as high as in rural areas.

Table 23 cross-classifies prevalence of diabetes by caste. As in other NCDs, the highest proportion of those suffering from diabetes were Others (over 47 per cent), followed by OBCs (about 38 per cent). Also, among Others, the proportion of diabetics was highest (about 19 per cent), as compared with barely 3.60 per cent among STs.

Table 23

Prevalence of Diabetes by Caste

\begin{tabular}{|c|c|c|c|c|c|}
\hline \multirow{2}{*}{$\begin{array}{c}\text { Whether Suffering } \\
\text { from Diabetes? }\end{array}$} & \multicolumn{4}{|c|}{ Caste } & \multirow{2}{*}{ Total } \\
\cline { 2 - 5 } & SCs & STs & OBCs & Others & \\
\hline \multirow{2}{*}{ No } & 20.53 & 4.86 & 37.15 & 37.46 & 100 \\
& $(88.77)$ & $(96.32)$ & $(83.66)$ & $(80.64)$ & $(84.01)$ \\
\hline \multirow{2}{*}{ Yes } & 13.79 & 0.98 & 37.96 & 47.27 & 100 \\
& $(11.11)$ & $(3.60)$ & $(15.93)$ & $(18.96)$ & $(15.65)$ \\
\hline \multirow{2}{*}{ Cured } & 6.85 & 1.03 & 45.82 & 46.30 & 100 \\
& $(0.12)$ & $(0.08)$ & $(0.41)$ & $(0.40)$ & $(0.34)$ \\
\hline \multirow{2}{*}{ Total } & 19.43 & 4.24 & 37.31 & 39.03 & 100 \\
& $(100)$ & $(100)$ & $(100)$ & $(100)$ & $(100)$ \\
\hline
\end{tabular}

Table 24

Prevalence of Cancer by Poverty Status

\begin{tabular}{|c|c|c|c|}
\hline \multirow{2}{*}{$\begin{array}{c}\text { Whether Suffering from } \\
\text { Cancer? }\end{array}$} & \multicolumn{2}{|c|}{ Below Poverty Line } & \multirow{2}{*}{ Total } \\
\cline { 2 - 3 } & No & Yes & 100 \\
No & 86.04 & 13.96 & $(98.62)$ \\
\hline \multirow{2}{*}{ Yes } & $(98.67)$ & $(98.33)$ & 100 \\
& 83.12 & 16.88 & $(1.24)$ \\
\hline \multirow{2}{*}{ Cured } & $(1.19)$ & $(1.49)$ & 100 \\
& 82.67 & 17.33 & $(0.14)$ \\
\hline \multirow{2}{*}{ Total } & $(0.14)$ & $(0.18)$ & 100 \\
& 86.00 & 14.00 & $(100)$ \\
\hline
\end{tabular}

\footnotetext{
${ }^{9}$ Bajpai et al. (2010) draw attention to rapid spread of diabetes to rural areas.
} 
As shown in Table 24, the vast majority of cancer cases (over 83 per cent) were reported among the non-poor. However, there was little difference in the proportions of the non-poor and poor reporting this condition. Table 25 further corroborates the association between cancer and (relative) affluence. Over 37 per cent of cancer cases were in the highest expenditure interval, followed by about 31 per cent in the next lower interval, as compared with barely 4 per cent in the lowest expenditure interval. The proportions of reported cancer cases among various expenditure groups, however, varied little.

Table 25

Prevalence of Cancer by Per Capita Monthly Expenditure (Rs)

\begin{tabular}{|c|c|c|c|c|c|}
\hline \multirow{2}{*}{$\begin{array}{c}\text { Whether Suffering } \\
\text { from Cancer? }\end{array}$} & \multicolumn{4}{|c|}{ Monthly Per Capita Expenditure (Rs) } & \multirow{2}{*}{ Total } \\
\cline { 2 - 5 } & $<300$ & $300-500$ & $500-1000$ & $>1000$ & 100 \\
\hline \multirow{2}{*}{ No } & 5.68 & 18.99 & 41.18 & 34.14 & $(98.47)$ \\
& $(99.14)$ & $(97.98)$ & $(98.97)$ & 37.39 & $(98.62)$ \\
\hline \multirow{2}{*}{ Yes } & 3.93 & 27.97 & 30.70 & $(1.35)$ & $(1.24)$ \\
& $(0.86)$ & $(1.81)$ & $(0.93)$ & 42.88 & 100 \\
Cured & 0.0 & 28.37 & 28.75 & $(0.18)$ & $(0.14)$ \\
& $(0.0)$ & $(0.21)$ & $(0.10)$ & 34.20 & 100 \\
Total & 5.65 & 19.12 & 41.03 & $(100)$ & $(100)$ \\
\hline
\end{tabular}

Table 26

Prevalence of Cancer by Location

\begin{tabular}{|c|c|c|c|c|}
\hline \multirow{2}{*}{$\begin{array}{c}\text { Whether Suffering } \\
\text { from Cancer? }\end{array}$} & \multicolumn{3}{|c|}{ Location } & \multirow{2}{*}{ Total } \\
\cline { 2 - 4 } & Rural & Urban & Urban Slums & \\
\hline \multirow{2}{*}{ No } & 69.77 & 28.94 & 1.29 & 100 \\
& $(98.55)$ & $(98.75)$ & $(99.23)$ & $(98.62)$ \\
\hline \multirow{2}{*}{ Yes } & 72.64 & 26.56 & 0.79 & 100 \\
& $(1.29)$ & $(1.14)$ & $(0.77)$ & $(1.24)$ \\
\hline \multirow{2}{*}{ Cured } & 77.35 & 22.65 & 0.0 & 100 \\
& $(0.16)$ & $(0.11)$ & $(0.0)$ & $(0.14)$ \\
\hline \multirow{2}{*}{ Total } & 69.81 & 28.91 & 1.28 & 100 \\
& $(100)$ & $(100)$ & $(100)$ & $(100)$ \\
\hline
\end{tabular}

Table 27

Prevalence of Cancer by Caste

\begin{tabular}{|c|c|c|c|c|c|}
\hline \multirow{2}{*}{$\begin{array}{c}\text { Whether Suffering } \\
\text { from Cancer? }\end{array}$} & \multicolumn{4}{|c|}{ Caste } & \multirow{2}{*}{ Total } \\
\cline { 2 - 5 } & SCs & STs & OBCs & Others & \\
\hline \multirow{2}{*}{ No } & 19.52 & 4.34 & 37.38 & 38.75 & 100 \\
& $(98.79)$ & $(99.72)$ & $(98.41)$ & $(98.62)$ & $(98.62)$ \\
\hline \multirow{2}{*}{ Yes } & 18.75 & 0.21 & 43.77 & 37.27 & 100 \\
& $(1.19)$ & $(0.06)$ & $(1.44)$ & $(1.19)$ & $(1.24)$ \\
\hline \multirow{2}{*}{ Cured } & 2.11 & 6.52 & 38.90 & 52.47 & 100 \\
& 0.02 & 0.22 & 0.15 & 0.19 & 0.14 \\
\hline \multirow{2}{*}{ Total } & 19.49 & 4.30 & 37.46 & 38.75 & 100 \\
& $(100)$ & $(100)$ & $(100)$ & $(100)$ & $(100)$ \\
\hline
\end{tabular}


Just under three quarters of the cancer cases were in rural areas, followed by a little over a quarter in urban areas. Going by caste affiliation in Table 27, about 43 per cent of cancer cases were OBCs and over 37 per cent were Others, as compared with barely 0.21 per cent among the STs-the most disadvantaged group.

In sum, there is ample evidence of strong links between NCDs and (relative) affluence. Besides, in some cases (e.g. heart disease, diabetes and cancer), the prevalence rates are high in rural areas.

\section{(c) Obesity and NCDs}

First, let us consider how prevalent obesity is and whether it is linked to affluence. Note that data used for constructing the body mass index (BMI) were collected for a relatively small sample $^{10}$. Following WHO norms, those with BMI between 25-30 are classified as overweight and those exceeding 30 are classified as obese.

Over 12 per cent of the sample individuals $>22$ years were overweight and over 3 per cent were obese. Under 50 per cent of the overweight and over 58 per cent of the obese were in urban areas. By contrast, the share of overweight was larger in rural areas (about 51 per cent) and that of obese was considerably lower (over 38 per cent). That vast majorities of both overweight and obese were non-poor is corroborated by Table 29 .

Table 28

BMI by Location

\begin{tabular}{|c|c|c|c|c|}
\hline Location/BMI Group & $0-25$ & $25-30$ & $>30$ & Total \\
\hline \multirow{2}{*}{ Rural } & $\begin{array}{c}89.23 \\
(75.09)\end{array}$ & $\begin{array}{c}9.05 \\
(50.96)\end{array}$ & $\begin{array}{c}1.72 \\
(38.36)\end{array}$ & $\begin{array}{c}100 \\
(70.88)\end{array}$ \\
\hline \multirow{2}{*}{ Urban } & 71.50 & 21.70 & 6.80 & 100 \\
& $(23.10)$ & $(46.90)$ & $(58.39)$ & $(27.21)$ \\
\hline \multirow{2}{*}{ Urban Slums } & 80.43 & 14.16 & 5.41 & 100 \\
& $(1.82)$ & $(2.14)$ & $(3.25)$ & $(1.90)$ \\
\hline \multirow{2}{*}{ Total } & 84.24 & 12.59 & 3.17 & 100 \\
& $(100)$ & $(100)$ & $(100)$ & $(100)$ \\
\hline
\end{tabular}

Table 29

BMI by Poverty Status

\begin{tabular}{|c|c|c|c|c|}
\hline $\begin{array}{c}\text { Poverty } \\
\text { Status/BMI Group }\end{array}$ & $0-25$ & $25-30$ & $>30$ & Total \\
\hline Non-Poor & $\begin{array}{c}82.11 \\
(75.98)\end{array}$ & $\begin{array}{c}14.18 \\
(87.79)\end{array}$ & $\begin{array}{c}3.71 \\
(91.20)\end{array}$ & $\begin{array}{c}100 \\
(77.95)\end{array}$ \\
\hline Poor & 91.76 & $\begin{array}{c}6.97 \\
(12.21)\end{array}$ & $\begin{array}{c}1.27 \\
(8.80)\end{array}$ & $\begin{array}{c}100 \\
(22.05)\end{array}$ \\
\hline Total & $(24.02)$ & 12.59 & 3.17 & 100 \\
& 84.24 & $(100)$ & $(100)$ & $(100)$ \\
\hline
\end{tabular}

\footnotetext{
${ }^{10} \mathrm{BMI}_{(\mathrm{kg} / \mathrm{m})}{ }^{2}=\left(\right.$ weight in $\mathrm{kg} /$ height in metres $\left.{ }^{2}\right)$.
} 
Let us now examine the links between obesity and NCDs.

Table 30

High Blood Pressure by BMI

\begin{tabular}{|c|c|c|c|c|c|}
\hline High BP/BMI Group & $<18$ & $18-25$ & $25-30$ & $>30$ & Total \\
\hline No & $\begin{array}{c}21.73 \\
(93.73)\end{array}$ & $\begin{array}{c}56.98 \\
(79.92)\end{array}$ & $\begin{array}{c}16.77 \\
(62.96)\end{array}$ & $\begin{array}{c}4.51 \\
(57.24)\end{array}$ & $\begin{array}{c}100 \\
(77.98)\end{array}$ \\
\hline \multirow{2}{*}{ Yes } & 4.82 & 48.87 & 34.46 & 11.86 & 100 \\
& $(5.90)$ & $(19.46)$ & $(36.73)$ & $(42.76)$ & $(22.01)$ \\
\hline \multirow{2}{*}{ Cured } & 13.97 & 72.62 & 13.41 & 0.0 & 100 \\
& $(0.37)$ & $(0.62)$ & $(0.31)$ & $(0.0)$ & $(0.47)$ \\
\hline \multirow{2}{*}{ Total } & 17.97 & 55.27 & 20.65 & 6.11 & 100 \\
& $(100)$ & $(100)$ & $(100)$ & $(100)$ & $(100)$ \\
\hline
\end{tabular}

While the share of overweight among those suffering from high blood pressure is high (over 34 per cent), that of obese is low (about 12 per cent). However, among the obese, the proportion reporting this condition was highest (about 43 per cent), followed by the overweight (about 37 per cent).

Over 59 per cent of those reporting heart disease had BMI in the (normal) range 18-25, over 20 per cent were overweight and barely over 8 per cent were obese. However, the proportion of obese reporting this condition was highest (over 13 per cent).

Table 31

Heart Disease by BMI

\begin{tabular}{|c|c|c|c|c|c|}
\hline $\begin{array}{c}\text { Heart Disease/ BMI } \\
\text { Group }\end{array}$ & $<18$ & $18-25$ & $25-30$ & $>30$ & Total \\
\hline No & $\begin{array}{c}19.10 \\
(93.41)\end{array}$ & $\begin{array}{c}54.76 \\
(89.84)\end{array}$ & $\begin{array}{c}20.59 \\
(90.53)\end{array}$ & $\begin{array}{c}5.54 \\
(86.53)\end{array}$ & $\begin{array}{c}100 \\
(90.45)\end{array}$ \\
\hline Yes & 12.15 & 59.09 & 20.23 & 8.53 & 100 \\
& $(6.01)$ & $(9.81)$ & $(9.0)$ & $(13.47)$ & $(9.15)$ \\
\hline Cured & 27.27 & 48.44 & 24.29 & 0.0 & 100 \\
& $(0.58)$ & $(0.35)$ & $(0.47)$ & $(0.0)$ & $(0.40)$ \\
\hline \multirow{2}{*}{ Total } & 18.50 & 55.13 & 20.57 & 5.79 & 100 \\
& $(100)$ & $(100)$ & $(100)$ & $(100)$ & $(100)$ \\
\hline
\end{tabular}

Table 32

Diabetes by BMI

\begin{tabular}{|c|c|c|c|c|c|}
\hline Diabetes/BMI Group & $<18$ & $18-25$ & $25-30$ & $>30$ & Total \\
\hline No & $\begin{array}{c}19.13 \\
(96.10)\end{array}$ & $\begin{array}{c}55.86 \\
(92.79)\end{array}$ & $\begin{array}{c}20.15 \\
(90.14)\end{array}$ & $\begin{array}{c}4.85 \\
(75.33)\end{array}$ & $\begin{array}{c}100 \\
(91.82)\end{array}$ \\
\hline \multirow{2}{*}{ Yes } & 8.72 & 48.43 & 24.33 & 18.51 & 100 \\
& $(3.76)$ & $(6.91)$ & $(9.35)$ & $(24.67)$ & $(7.89)$ \\
\hline \multirow{2}{*}{ Cured } & 8.17 & 56.73 & 35.11 & 0.0 & 100 \\
& $(0.13)$ & $(0.30)$ & $(0.51)$ & $(0.0)$ & $(0.30)$ \\
\hline \multirow{2}{*}{ Total } & 18.28 & 55.28 & 20.53 & 5.92 & 100 \\
& $(100)$ & $(100)$ & $(100)$ & $(100)$ & $(100)$ \\
\hline
\end{tabular}


Again a strong link between overweight or obesity and diabetes is not discernible, as the largest proportion of diabetics is in the normal range of BMI (18-25). However, of the obese, nearly a quarter reported this condition-by far the highest proportion of different ranges of BMI.

As cancer is unlikely to be associated with being overweight or obese, any further comment is unnecessary.

We also investigated the link between eating out and overweight and obesity. To avoid cluttering the text, the details are eschewed. The main finding is that in the highest expenditure interval for eating out (Rs 500 per month) the proportion of overweight is over 22 per cent) as against under 12 per cent among those who did not eat out at all. Also, the proportion of obese in this expenditure interval (0ver 6 per cent) is more than twice that in the households that did not eat our. So the links between eating out and overweight/obesity and their implications for NCDs are not clear-cut.

In sum, while being overweight or obese enhances the risk of NCDs, further investigation is necessary to isolate the effects of the former.

\section{Determinants of NCDs}

\section{(a) Methodology}

As the cross-classifications cannot isolate the causal relationships, we use two econometric specifications for isolating the effects of household, village and prices of various food commodities/nutrients on the occurrence of specific NCDs.

In the first probit specification, we examine the factors underlying occurrence of high blood pressure, heart disease, diabetes and cancer.

In a binary response model (whether an individual suffers from, say, heart disease), interest lies primarily in the response probability

$P(y=1 \mid x)=P\left(y=1 \mid x_{1}, x_{2}, \ldots \ldots . . . x_{k}\right), \ldots$

where $\mathbf{x}$ denotes the full set of explanatory variables.

Consider a class of binary response models of the form

$\mathrm{P}(\mathrm{y}=1 \mid \mathbf{x})=\mathrm{G}\left(\beta_{0}+\mathbf{x} \boldsymbol{\beta}\right)$,

where $G$ is a function taking on values strictly between zero and one: $0<G(\mathbf{z})<1$, for all real numbers $\mathrm{z}$.

In the probit model, $\mathrm{G}$ is the standard normal cumulative distribution function (cdf) ${ }^{11}$.

\footnotetext{
${ }^{11}$ For further details, see Wooldridge (2006).
} 
In another specification our dependent variable is average incidence of NCDs (i.e. number of household members suffering from high blood pressure or heart disease or diabetes or cancer/household size). As these averages cluster around zero or small values in the range 0 1 , a tobit specification is employed. In a variant, we add up the number of ailments reported by household members divided by household size. Some illustrative results are given.

Typically, the tobit model expresses the observed response, y, in terms of an underlying latent variable

$$
\begin{aligned}
& \mathrm{y}^{*}=\beta_{0}+\mathbf{x} \boldsymbol{\beta}+\mu, \mu \mid \mathbf{x} \square \operatorname{Normal}\left(0, \sigma^{2}\right) \\
& y=\max \left(0, y^{*}\right) \ldots \ldots . . .(3)
\end{aligned}
$$

The latent variable $\mathrm{y}^{*}$ satisfies the classical linear model assumptions; in particular, it has a normal homoscedastic distribution with a linear conditional mean. Equation (3) implies that the observed variable y equals $\mathrm{y}^{*}$ when $\mathrm{y}^{*} \geq 0$, but $\mathrm{y}=0$ when $\mathrm{y}^{*}<0$. Since $\mathrm{y}^{*}$ is normally distributed, $\mathrm{y}$ has a continuous distribution over strictly positive values. ${ }^{12}$

\section{(b) Results}

The probit results are given for each of the four diseases. Table 33 throws light on the factors associated with high blood pressure (HBP). As this is essentially a reduced form relation, and the links between nutritional changes and disease outcomes are ambiguous, any definitive conclusions about how price effects impinge on the risk of a disease must be avoided. Our results are reported separately for rural and urban areas.

\section{(b.1) High Blood Pressure}

The main findings for the rural areas are:

- Age and (incidence of) HBP are positively related. However, this relationship weakens with advancing age.

- Women are more likely to suffer from HBP than men.

- Completed years of education and HBP are positively related but this relationship weakens at higher levels of education (or more years of education).

- There is no association between caste and HBP.

- (Predicted) log of per capita expenditure and HBP are negatively related but this relationship weakens with higher levels of expenditure.

- There is no relationship between distance to the nearest hospital and HBP.

- The price of rice and risk of HBP are positively related, as also those of wheat and other cereals. However, the price of cereal products and HBP are inversely relared. ${ }^{13}$

- The price of gur and HBP are inversely related.

- The price of edible oil and milk are inversely related to the risk of HBP.

- Prices of vegetables, and salt and spices are also inversely related to the risk of HBP.

- Higher prices of tea and coffee are associated with higher risks of HBP.

- Also, higher prices of fruits and nuts are associated with significantly higher risks of HBP.

\footnotetext{
${ }^{12}$ For further details, see Wooldridge (2006).

${ }^{13}$ Note that prices and expenditure per capita are all in logs.
} 
Affluence, Obesity and Non-Communicable Diseases in India

Table: 33 Factors Associated with High Blood Pressure in India

\begin{tabular}{|c|c|c|c|c|}
\hline \multirow{2}{*}{$\begin{array}{l}\text { Sample } \\
\text { Explanatory variables }\end{array}$} & \multicolumn{2}{|c|}{ Rural India } & \multicolumn{2}{|c|}{ Urban India } \\
\hline & Coeff.(z-value) & $\begin{array}{c}\text { Marginal effect } \\
\text { (z-value) }\end{array}$ & Coeff.(z-value) & $\begin{array}{l}\text { Marginal effect } \\
\text { (z-value) }\end{array}$ \\
\hline Age & $0.072^{\star \star *}(12.20)$ & $0.0002^{\star \star \star}(8.36)$ & $0.088^{\star \star \star}(13.60)$ & $0.0006^{\star \star \star}(9.43)$ \\
\hline Square of age & $-0.0005^{\star \star \star}(-8.04)$ & $-0.000001^{\star * \star(-7.96)}$ & $-0.001^{\star \star *}(-8.93)$ & $-0.000003^{\star \star \star}(-9.35)$ \\
\hline Gender & $0.335^{\star \star \star}(8.21)$ & $0.0009^{\star \star \star}(5.14)$ & $0.237^{\star \star \star}(6.04)$ & $0.0015^{\star \star \star}(4.75)$ \\
\hline Years of education & $0.045^{\star \star \star}(3.42)$ & $0.0001^{\star \star \star}(3.07)$ & $0.036^{\star \star \star}(3.04)$ & $0.0002^{\star \star \star}(2.86)$ \\
\hline Square of years of education & $-0.003^{\star \star \star}(-2.58)$ & $-0.000007 * *(-2.35)$ & $-0.003^{\star \star \star}(-3.80)$ & $-0.00002^{\star \star *}(-3.28)$ \\
\hline OBC & $0.057(0.53)$ & $0.0002(0.49)$ & $-0.445^{\star \star}(-1.98)$ & $-0.0016^{\star \star *}(-3.44)$ \\
\hline SC & $-0.005(-0.09)$ & $0.0000(-0.09)$ & $-0.050(-0.84)$ & $-0.0003(-0.85)$ \\
\hline ST & $0.061(1.05)$ & $0.0002(0.99)$ & $0.021(0.37)$ & $0.0001(0.37)$ \\
\hline Log PCME (IV) & $-2.666^{\star \star}(-2.37)$ & $-0.0070^{\star *}(-2.14)$ & $-2.608^{\star \star \star}(-2.57)$ & $-0.0163^{\star \star}(-2.33)$ \\
\hline Square of Log PCME (IV) & $0.243^{\star \star \star}(2.83)$ & $0.0006^{\star \star}(2.47)$ & $0.213^{\star \star \star}(2.83)$ & $0.0013^{\star \star}(2.52)$ \\
\hline Village distance from nearest hospital & $0.000(0.57)$ & $0.0000(0.57)$ & & \\
\hline Metro & & & $0.050(0.91)$ & $0.0003(0.88)$ \\
\hline Slum & & & $-0.077(-0.95)$ & $-0.0004(-1.03)$ \\
\hline Log Price of rice at PSU level & $0.327^{\star \star \star}(3.22)$ & $0.0009^{\star * \star}(2.85)$ & $0.254^{\star \star}(2.32)$ & $0.0016^{\star \star}(2.19)$ \\
\hline Log Price of wheat at PSU level & $0.551^{\star \star \star}(5.35)$ & $0.0014^{\star \star \star}(4.09)$ & $-0.068(-0.57)$ & $-0.0004(-0.57)$ \\
\hline Log Price of sugar at PSU level & $0.097(0.45)$ & $0.0003(0.45)$ & $-0.682^{\star \star \star}(-2.97)$ & $-0.0043^{\star \star \star}(-2.74)$ \\
\hline Log Price of other cereals at PSU level & $0.084^{*}(1.67)$ & $0.0002^{\mathrm{w}}(1.62)$ & $0.165^{\star \star \star}(3.27)$ & $0.0010^{\star \star \star}(2.95)$ \\
\hline Log Price of cereal product at PSU level & $-0.218^{\star \star}(-2.12)$ & $-0.0006^{* *}(-2.04)$ & $-0.030(-0.29)$ & $-0.0002(-0.29)$ \\
\hline Log Price of pulses at PSU level & $0.012(0.10)$ & $0.0000(0.1)$ & $0.170^{w}(1.59)$ & $0.0011 w(1.55)$ \\
\hline Log Price of meat at PSU level & $-0.017(-0.24)$ & $0.0000(-0.24)$ & $0.129 *(1.86)$ & $0.0008^{\star}(1.81)$ \\
\hline Log Price of gur at PSU level & $-0.232^{\star \star \star}(-3.20)$ & $-0.0006^{\star \star \star}(-2.85)$ & $-0.233^{\star \star \star}(-3.37)$ & $-0.0015^{\star \star \star}(-3.06)$ \\
\hline Log Price ofr edible-oil at PSU level & $0.943^{\star \star \star}(3.82)$ & $0.0025^{\star \star \star}(3.28)$ & $1.002^{\star \star \star}(4.38)$ & $0.0063^{\star \star \star}(3.72)$ \\
\hline Log Price of eggs at PSU level & $-0.221 *(-1.83)$ & $-0.0006 *(-1.76)$ & $-0.422^{\star \star \star}(-2.60)$ & $-0.0026^{\star \star}(-2.44)$ \\
\hline Log Price of milk at PSU level & $-0.795^{\star \star \star}(-5.12)$ & $-0.0021^{\star \star *}(-4.03)$ & $-0.547^{\star \star \star}(-3.48)$ & $-0.0034^{\star \star \star}(-3.1)$ \\
\hline Log Price of milk-products at PSU level & $0.030(1.07)$ & $0.0001(1.05)$ & $0.013(0.38)$ & $0.0001(0.38)$ \\
\hline Log d Price of vegetables at PSU level & $-0.238^{\star \star}(-2.52)$ & $-0.0006^{* *(-2.34)}$ & $0.057(0.72)$ & $0.0004(0.71)$ \\
\hline Log Price of salt-spices at PSU level & $-0.725^{\star \star \star(-3.64)}$ & $-0.0019^{\star \star \star}(-3.13)$ & $-1.178^{\star \star \star}(-6.49)$ & $-0.0074^{\star \star \star}(-4.74)$ \\
\hline Log Price of tea-coffee at PSU level & $0.279 *(2.52)$ & $0.0007^{\star \star}(2.39)$ & $0.070(0.51)$ & $0.0004(0.51)$ \\
\hline Log Price of paan-tobacco at PSU level & $0.023(1.24)$ & $0.0001(1.21)$ & $-0.241^{\star \star \star}(-5.45)$ & $-0.0015^{\star \star \star}(-4.36)$ \\
\hline Log Price of fruits-nuts at PSU level & $0.181^{w}(1.61)$ & $0.0005^{w}(1.57)$ & $0.211 *(1.83)$ & $0.0013^{\star}(1.77)$ \\
\hline Constant & $2.178(0.55)$ & & $7.733^{\star \star}(2.08)$ & \\
\hline Number of observations & 62594 & & 37128 & \\
\hline LR chi-square & $1936.90^{\star \star \star}$ & & 2100.11 *** & \\
\hline Pseudo R-square & 0.2726 & & 0.2705 & \\
\hline Log likelihood & -2584.4075 & & -2831.758 & \\
\hline Predicted probability & & 0.00078 & & 0.002 \\
\hline
\end{tabular}

*** Significant at the $1 \%$ level, ** significant at the $5 \%$ level, * significant at the $10 \%$ level, w weakly significant at the $10 \%$ level. 
Let us now compare these with the results for the urban areas. Many of the results are similar to those obtained for the rural areas. Let us first note the similarities.

- Age, sex and education have similar associations with the risk of HBP.

- Expenditure and risk of HBP are also similarly related to this condition.

- The price effects vary between the rural and urban samples. Let us first consider those that are similar. These include prices of rice, other cereals, gur, milk, salt and spices, and fruits and nuts. Different results are obtained for the prices of sugar, pulses, meat, edible oil, eggs, and paan and tobacco.

- The risk of HBP is higher in the metros relative to other urban areas excluding urban slums.

\section{Marginal Effects}

To avoid repetition, our comments are confined to economically significant marginal effects and any differences between rural and urban results.

- In the rural sample, most of the effects are small. However, since marginal effects depend on the unit of measurement, we cannot assess whether some are larger than others except to note whether the magnitudes involved are large. Subject to this caveat, the effect of expenditure is not-so-small. However, that of the square of expenditure is. Among the food prices, the effects of wheat, edible oil, milk, and salt and spices are not-so-small while others are.

- In the urban sample, many of the effects are slightly larger. These include gender, OBCs, expenditure and its square, and among the food prices, rice, sugar, other cereals, gur, edible oil, eggs, milk, vegetables, tea and coffee, and paan and tobacco.

\section{(b.2) Heart Disease}

We refer here to the probit results in Table 34, beginning with the rural sample.

- The risk of heart disease and age are positively related but this relationship weakens with advancing age.

- Women are more prone to heart disease.

- The risk of heart disease rises with years of education but at a diminishing rate.

- The STs are more prone to heart disease relative to Others.

- The risk of heart disease diminishes with higher per capita expenditure but with a weakening of this relationship at higher levels of per capita expenditure.

- The price effects are varied: sugar, edible oil, milk, and tea and coffee prices are associated with higher risks of heart disease while those of other cereals, eggs, and salt and spices are associated with lower risks of this condition.

Let us now compare these with the urban results.

- As in the rural sample, age is positively related to the risk of heart disease while the square of age is negatively related to it.

- Somewhat surprisingly, neither expenditure nor its square are significantly associated with the risk of heart disease.

- Among the food prices, there are sign reversals and other differences. Let us first consider the sign reversals. Sugar, milk, and tea and coffee have a negative association with the risk of heart disease. Other price variables that have significant associations with this condition include cereal products (negative), vegetables (positive), paan and tobacco (positive) and fruits and nuts (negative). 
Table 34 Factors Associated with Heart Disease in India

\begin{tabular}{|c|c|c|c|c|}
\hline Sample & Rura & ndia & & India \\
\hline Explanatory variables & Coeff.(z-value) & $\begin{array}{c}\text { Marginal effect } \\
\text { (z-value) }\end{array}$ & Coeff.(z-value) & $\begin{array}{c}\text { Marginal effect } \\
\text { (z-value) }\end{array}$ \\
\hline Age & $0.043^{\star \star *}(7.94)$ & $0.0002^{\star \star \star}(9.07)$ & $0.039^{\star \star \star}(6.36)$ & $0.0003^{\star \star \star}(8.01)$ \\
\hline Square of age & $-0.0003^{\star \star \star}(-5.30)$ & $-0.000002^{\star \star \star}(-5.88)$ & $-0.0002^{\star \star}(-2.53)$ & $-0.000001^{\star \star \star}(-2.87)$ \\
\hline Gender & $0.112^{\star \star}(2.21)$ & $0.0006^{\star *}(2.17)$ & $-0.051(-0.95)$ & $-0.0004(-0.94)$ \\
\hline Years of education & $0.057^{\star * \star(3.32)}$ & $0.0003^{\star \star *}(3.25)$ & $-0.004(-0.24)$ & $0.0000(-0.24)$ \\
\hline Square of years of education & $-0.005^{\star \star \star}(-3.41)$ & $-0.00002^{\star \star \star}(-3.3)$ & $0.000(-0.02)$ & $0.0000(-0.02)$ \\
\hline OBC & $-0.093(-0.65)$ & $-0.0004(-0.73)$ & $-0.112(-0.51)$ & $-0.0007(-0.6)$ \\
\hline SC & $0.037(0.52)$ & $0.0002(0.52)$ & $-0.012(-0.14)$ & $-0.0001(-0.14)$ \\
\hline ST & $0.146 *(1.96)$ & $0.0008^{*}(1.77)$ & $-0.036(-0.45)$ & $-0.0002(-0.45)$ \\
\hline Log PCME (IV) & $-2.497 *(-1.95)$ & $-0.0124^{\star}(-1.9)$ & $-1.148(-0.84)$ & $-0.0079(-0.84)$ \\
\hline Square of Log PCME (IV) & $0.211^{\star \star(2.13)}$ & $0.0010 \star \star(2.07)$ & $0.099(0.98)$ & $0.0007(0.97)$ \\
\hline Village distance from nearest hospital & $-0.001(-1.28)$ & $0.0000(-1.27)$ & & \\
\hline Metro & & & $-0.044(-0.55)$ & $-0.0003(-0.56)$ \\
\hline Slum & & & $0.038(0.33)$ & $0.0003(0.31)$ \\
\hline Log Price of rice at PSU level & $-0.036(-0.27)$ & $-0.0002(-0.27)$ & $0.120(0.78)$ & $0.0008(0.78)$ \\
\hline Log Price of wheat at PSU level & $0.172(1.28)$ & $0.0009(1.27)$ & $0.155(0.87)$ & $0.0011(0.87)$ \\
\hline Log Price of sugar at PSU level & $0.522 *(1.75)$ & $0.0026^{*}(1.73)$ & $-0.877^{\star \star \star}(-2.94)$ & $-0.0061^{\star \star \star}(-2.84)$ \\
\hline Log Price of other cereals at PSU level & $-0.231^{* * *(-3.45)}$ & $-0.0011^{\star \star \star}(-3.32)$ & $0.032(0.46)$ & $0.0002(0.46)$ \\
\hline Log Price of cereal product at PSU level & $-0.166(-1.43)$ & $-0.0008(-1.42)$ & $-0.301^{\star \star}(-2.22)$ & $-0.0021^{\star \star}(-2.16)$ \\
\hline Log Price of pulses at PSU level & $-0.162(-1.11)$ & $-0.0008(-1.1)$ & $-0.195(-1.45)$ & $-0.0013(-1.43)$ \\
\hline Log Price of meat at PSU level & $-0.067(-0.72)$ & $-0.0003(-0.72)$ & $-0.054(-0.59)$ & $-0.0004(-0.59)$ \\
\hline Log Price of gur at PSU level & $-0.112(-1.21)$ & $-0.0006(-1.2)$ & $0.091(1.19)$ & $0.0006(1.18)$ \\
\hline Log Price of edible-oil at PSU level & $0.692^{\star \star}(2.12)$ & $0.0034^{\star *}(2.09)$ & $-0.057(-0.19)$ & $-0.0004(-0.19)$ \\
\hline Log Price of eggs at PSU level & $-0.288^{\star \star}(-2.00)$ & $-0.0014^{\star \star}(-1.99)$ & $0.045(0.21)$ & $0.0003(0.21)$ \\
\hline Log Price of milk at PSU level & $0.303 *(1.70)$ & $0.0015 *(1.68)$ & $-0.355^{\star}(-1.67)$ & $-0.0025^{\star}(-1.65)$ \\
\hline Log Price of milk-products at PSU level & $0.030(0.87)$ & $0.0002(0.87)$ & $0.018(0.35)$ & $0.0001(0.35)$ \\
\hline Log Price of vegetables at PSU level & $-0.100(-0.90)$ & $-0.0005(-0.9)$ & $0.297^{\star \star \star}(3.10)$ & $0.0021^{* \star \star}(2.95)$ \\
\hline Log Price of salt-spices at PSU level & $-0.626^{\star \star}(-2.40)$ & $-0.0031^{\star *}(-2.36)$ & $0.601 * *(2.36)$ & $0.0042^{* \star}(2.3)$ \\
\hline Log Price of tea-coffee at PSU level & $0.288^{\star \star}(2.13)$ & $0.0014 * *(2.12)$ & $-0.399 \star *(-2.17)$ & $-0.0028^{\star \star}(-2.11)$ \\
\hline Log Price ofr paan-tobacco at PSU level & $0.034(1.47)$ & $0.0002(1.46)$ & $0.166^{\star \star \star}(2.81)$ & $0.0011^{\star \star \star}(2.7)$ \\
\hline Log Price of fruits-nuts at PSU level & $-0.065(-0.50)$ & $-0.0003(-0.5)$ & $-0.530 * \star *(-3.63)$ & $-0.0037^{\star \star}(-3.42)$ \\
\hline Constant & $3.097(0.67)$ & & $2.682(0.54)$ & \\
\hline Number of observations & 62594 & & 37128 & \\
\hline LR chi-square & $362.82^{\star \star \star}$ & & $458.81^{\star \star \star}$ & \\
\hline Pseudo R-square & 0.1159 & & 0.1548 & \\
\hline Log likelihood & -1384.3565 & & -1252.6023 & \\
\hline Predicted probability & & 0.0015 & & 0.0022 \\
\hline
\end{tabular}

*** Significant at the $1 \%$ level, ${ }^{* *}$ significant at the $5 \%$ level, * significant at the $10 \%$ level, w weakly significant at the $10 \%$ level. 
In an alternative specification, we use height as a measure of cumulative nutritional advantage. Although it has the expected negative sign and the coefficient is significant, the marginal effect is small. Other marginal effects are mostly unaffected ${ }^{14}$.

\section{Marginal Effects}

- In the rural sample, many of the effects are small. Some of the price effects that are not so small include other cereals, gur, eggs, milk, vegetables, and salt and spices.

- While milk, vegetable, and salt and spices price effects are not so small in the urban sample, those of sugar, cereal products tea and coffee, paan and tobacco and fruits and nuts are also not- so- small.

\section{(b.3) Diabetes}

Our comments are based on the results in Table 35, beginning with those obtained from the rural sample.

- While age is positively related to the risk of diabetes, this relationship weakens with advancing age.

- Women are less prone to diabetes.

- The risk of diabetes rises with years of education but at a slower rate.

- Higher expenditure reduces the risk of diabetes but at a slower rate.

- The price effects are varied: the prices of rice, sugar, other cereals, edible oils, salt and spices, tea and coffee and paan and tobacco are positively linked to the risk of diabetes while those of eggs, and salt and spices are negatively linked to this risk.

Let us now turn to the urban results.

- Age and age squared, and years of education and square of years of education have similar relationships with the risk of diabetes in the urban areas.

- Expenditure and its square are not related to this risk.

- OBCs are less prone to diabetes relative to Others.

- Urban slums are less likely to have diabetes than other urban areas.

- Among food prices, there are some differences. For example, prices of cereal products, meat, gur, milk products, and fruits and nuts have significant effects only in the urban sample while those of sugar, and paan and tobacco have significant effects in both samples.

\section{Marginal Effects}

As shown in Table 35, all marginal effects are very small in both rural and urban samples and thus no further comment is necessary.

\section{(b.4) Cancer}

Table 36 gives the probit results for cancer. We will comment first on the rural results.

- The risk of cancer rises with age.

- The STs are more prone to this risk than Others.

- Education has a weakly significant negative relationship with cancer.

- There is no relationship between expenditure and cancer risk.

\footnotetext{
${ }^{14}$ This is meant to illustrate the importance of cumulative nutritional status in limiting vulnerability to different NCDs. The case considered is not intended to suggest that height matters only in limiting the risk of heart disease. For details, see Table A.2.
} 
- The price effects vary: the price of milk is positively related to the risk of cancer while those of other cereals, pulses, vegetables, and paan and tobacco are negatively related to this risk.

Table 35: Factors Associated with Diabetes in India

\begin{tabular}{|c|c|c|c|c|}
\hline Sample & & India & Urba & ndia \\
\hline Explanatory variables & Coeff.(z-value) & $\begin{array}{c}\text { Marginal effect } \\
\text { (z-value) }\end{array}$ & Coeff.(z-value) & $\begin{array}{c}\text { Marginal effect } \\
\text { (z-value) }\end{array}$ \\
\hline Age & $0.086^{\star \star \star}(8.99)$ & $0.00004^{\star \star \star}(3.76)$ & $0.143^{\star \star \star}(11.88)$ & $0.0001 * \star *(3.05)$ \\
\hline Square of age & $-0.001^{\star * \star}(-6.67)$ & $-0.0000003^{\star \star \star}(-3.90)$ & $-0.001^{\star \star \star}(-9.59)$ & $-0.000001^{\star \star \star}(-3.14)$ \\
\hline Gender & $-0.123^{\star *}(-2.34)$ & $-0.00006^{\star}(-1.81)$ & $-0.011(-0.22)$ & $0.0000(-0.21)$ \\
\hline Years of education & $0.072^{\star \star \star}(4.05)$ & $0.00004^{\star *}(2.36)$ & $0.045^{\star \star \star}(3.01)$ & $0.00003^{\star *}(1.97)$ \\
\hline Square of years of education & $-0.006^{\star \star \star}(-4.23)$ & $-0.000003^{\star \star}(-2.34)$ & $-0.002^{\star \star}(-2.37)$ & $-0.000001^{*}(-1.69)$ \\
\hline $\mathrm{OBC}$ & $-0.293(-1.36)$ & $-0.0001 *(-1.78)$ & $-0.660 *(-1.79)$ & $-0.0001^{* *}(-2.27)$ \\
\hline SC & $0.070(0.94)$ & $0.00004(0.87)$ & $-0.056(-0.75)$ & $0.0000(-0.74)$ \\
\hline ST & $0.049(0.60)$ & $0.00003(0.57)$ & $-0.045(-0.63)$ & $0.0000(-0.61)$ \\
\hline Log PCME (IV) & $-3.320 * *(-2.22)$ & $-0.00173^{\star}(-1.67)$ & $1.691(1.02)$ & $0.0010(0.99)$ \\
\hline Square of Log PCME (IV) & $0.297^{\star \star \star}(2.63)$ & $0.00015^{*}(1.84)$ & $-0.104(-0.85)$ & $-0.0001(-0.84)$ \\
\hline Village distance from nearest hospital & $-0.001(-0.55)$ & $0.00000(-0.54)$ & & \\
\hline Metro & & & $-0.063(-0.96)$ & $0.0000(-0.93)$ \\
\hline Slum & & & $-0.223^{\star \star}(-1.97)$ & $-0.0001^{*}(-1.85)$ \\
\hline Log Price of rice at PSU level & $0.465^{\star \star \star}(3.29)$ & $0.00024^{\star \star}(2.21)$ & $0.177(1.33)$ & $0.0001(1.17)$ \\
\hline Log Price of wheat at PSU level & $-0.160(-1.21)$ & $-0.00008(-1.14)$ & $-0.118(-0.81)$ & $-0.0001(-0.78)$ \\
\hline Log Price of sugar at PSU level & $0.883^{\star \star \star}(2.84)$ & $0.00046^{\star \star}(2.09)$ & $-0.477^{\star}(-1.71)$ & $-0.0003(-1.43)$ \\
\hline Log Price of other cereals at PSU level & $0.119 *(1.71)$ & $0.00006^{w}(1.50)$ & $0.331^{\star \star \star}(5.32)$ & $0.0002^{\star *}(2.31)$ \\
\hline Log Price ofr cereal product at PSU level & $-0.128(-0.94)$ & $-0.00007(-0.91)$ & $0.108(0.88)$ & $0.0001(0.83)$ \\
\hline Log Price of pulses at PSU level & $0.148(1.05)$ & $0.00008(0.99)$ & $0.488^{* \star *}(3.39)$ & $0.0003^{\star \star}(2.07)$ \\
\hline Log Price of meat at PSU level & $0.085(0.89)$ & $0.00004(0.85)$ & $0.179 * \star(2.14)$ & $0.0001^{*}(1.65)$ \\
\hline Log Price of gur at PSU level & $0.124(1.43)$ & $0.00006(1.30)$ & $-0.151 *(-1.79)$ & $-0.0001(-1.48)$ \\
\hline Log Price of edible-oil at PSU level & $0.637 *(2.03)$ & $0.00033^{\star}(1.67)$ & $0.070(0.24)$ & $0.0000(0.24)$ \\
\hline Log Price of eggs at PSU level & $-0.330 * *(-2.08)$ & $-0.00017^{\star}(-1.73)$ & $0.173(0.90)$ & $0.0001(0.84)$ \\
\hline Log Price of milk at PSU level & $0.093(0.51)$ & $0.00005(0.50)$ & $0.107(0.56)$ & $0.0001(0.55)$ \\
\hline Log d Price of milk-products at PSU level & $0.001(0.02)$ & $0.00000(0.02)$ & $-0.044(-1.00)$ & $0.0000(-0.94)$ \\
\hline Log Price of vegetables at PSU level & $-0.033(-0.29)$ & $-0.00002(-0.28)$ & $-0.179 *(-1.66)$ & $-0.0001^{*}(-1.41)$ \\
\hline Log Price of salt-spices at PSU level & $-1.635^{\star \star \star}(-5.04)$ & $-0.00085^{\star \star \star}(-2.58)$ & $-1.382^{\star \star \star}(-6.09)$ & $-0.0008^{* *}(-2.37)$ \\
\hline Log Price of tea-coffee at PSU level & $0.286 *(1.84)$ & $0.00015^{w}(1.61)$ & $0.268(1.47)$ & $0.0002(1.30)$ \\
\hline Log Price of paan-tobacco at PSU level & $0.058 *(2.35)$ & $0.00003^{*}(1.79)$ & $-0.119 *(-2.18)$ & $-0.0001 *(-1.70)$ \\
\hline Log Price of fruits-nuts at PSU level & $0.164(1.16)$ & $0.00009(1.08)$ & $0.390^{\star \star \star}(2.73)$ & $0.0002^{*}(1.89)$ \\
\hline Constant & $2.970(0.56)$ & & $-11.171^{*}(-1.86)$ & \\
\hline Number of observations & 62594 & & 37128 & \\
\hline LR chi-square & $1260.77^{\star \star \star}$ & & $1379.92^{\star \star \star}$ & \\
\hline Pseudo R-square & 0.3040 & & 0.2760 & \\
\hline Log likelihood & -1443.5336 & & -1810.0141 & \\
\hline Predicted probability & & 0.0001 & & 0.0001 \\
\hline
\end{tabular}

*** Significant at the $1 \%$ level, ** significant at the $5 \%$ level, * significant at the $10 \%$ level, w weakly significant at the $10 \%$ level. 
Table 36 Factors Associated with Cancer in India

\begin{tabular}{|c|c|c|c|c|}
\hline Sample & & & Urba & India \\
\hline Explanatory variables & Coeff.(z-value) & $\begin{array}{c}\text { Marginal effect } \\
\text { (z-value) }\end{array}$ & Coeff.(z-value) & $\begin{array}{c}\text { Marginal effect } \\
\text { (z-value) }\end{array}$ \\
\hline Age & $0.025^{\star \star}(2.00)$ & $0.00001 *(1.75)$ & $0.047^{\star \star}(2.82)$ & $0.00001^{* *}(2.06)$ \\
\hline Square of age & $0.000(-0.90)$ & $0.0000(-0.91)$ & $-0.0003^{\star}(-1.85)$ & $-0.0000001^{*}(-1.69)$ \\
\hline Gender & $-0.023(-0.17)$ & $0.0000(-0.17)$ & $-0.157(-1.12)$ & $0.0000(-0.96)$ \\
\hline Years of education & $-0.072(-1.52)$ & $0.0000(-1.31)$ & $-0.034(-0.78)$ & $0.0000(-0.7)$ \\
\hline Square of years of education & $0.005(1.44)$ & $0.0000(1.25)$ & $-0.001(-0.26)$ & $0.0000(-0.26)$ \\
\hline $\mathrm{OBC}$ & Omitted & Omitted & $-0.365(-0.63)$ & $-0.0001(-1.07)$ \\
\hline SC & $0.071(0.36)$ & $0.0000(0.35)$ & $-0.019(-0.11)$ & $0.0000(-0.11)$ \\
\hline ST & $0.375^{\star}(1.87)$ & $0.0001(1.25)$ & $-0.326^{*}(-1.67)$ & $-0.0001(-1.24)$ \\
\hline Log PCME (IV) & $-3.398(-1.21)$ & $-0.0009(-1.08)$ & $-5.625^{\star \star}(-2.55)$ & $-0.0017 \mathrm{w}(-1.58)$ \\
\hline Square of Log PCME (IV) & $0.247(1.10)$ & $0.0001(1.00)$ & $0.424^{\star \star}(2.46)$ & $0.0001^{w}(1.55)$ \\
\hline Village distance from nearest hospital & $0.002(0.63)$ & $0.0000(0.59)$ & & \\
\hline Metro & & & $-0.198(-0.96)$ & $-0.0001(-0.95)$ \\
\hline Slum & & & $-0.370(-1.11)$ & $-0.0001(-1.35)$ \\
\hline Log Price of rice at PSU level & $0.247(0.75)$ & $0.0001(0.70)$ & $-0.525(-1.31)$ & $-0.0002(-1.09)$ \\
\hline Log Price of wheat at PSU level & $0.332(0.68)$ & $0.0001(0.68)$ & $1.396^{\star \star}(2.51)$ & $0.0004 \mathrm{w}(1.63)$ \\
\hline Log Price of sugar at PSU level & $-0.924(-1.08)$ & $-0.0002(-0.97)$ & $-2.206^{\star \star \star}(-2.83)$ & $-0.0007^{\star}(-1.66)$ \\
\hline Log Price of other cereals at PSU level & $-0.364^{*}(-1.66)$ & $-0.0001(-1.42)$ & $-0.083(-0.41)$ & $0.0000^{w}(-0.4)$ \\
\hline Log Price of cereal product at PSU level & $0.398(1.43)$ & $0.0001(1.23)$ & $0.706^{\star \star \star}(2.63)$ & $0.0002(1.54)$ \\
\hline Log Price of pulses at PSU level & $-0.926^{\star *}(-2.07)$ & $-0.0002^{\mathrm{w}}(-1.63)$ & $-0.255(-0.70)$ & $-0.0001(-0.67)$ \\
\hline Log Price of meat at PSU level & $0.260(0.90)$ & $0.0001(0.83)$ & $0.673^{\star \star}(2.16)$ & $0.0002 \mathrm{w}(1.57)$ \\
\hline Log Price of gur at PSU level & $-0.427(-1.40)$ & $-0.0001(-1.23)$ & $-0.164(-0.70)$ & $-0.0001(-0.65)$ \\
\hline Log Price of edible-oil at PSU level & $-0.131(-0.15)$ & $0.0000(-0.15)$ & $-0.525(-0.65)$ & $-0.0002^{*}(-0.62)$ \\
\hline Log Price of eggs at PSU level & $-0.363(-1.11)$ & $-0.0001(-1.02)$ & $0.389(0.76)$ & $0.0001(0.72)$ \\
\hline Log Price of milk at PSU level & $1.289 * \star \star(2.75)$ & $0.0003^{*}(1.85)$ & $-0.730(-1.25)$ & $-0.0002^{\star}(-1.11)$ \\
\hline Log Price of milk-products at PSU level & $0.128(1.08)$ & $0.0000(1.03)$ & $-0.099(-0.74)$ & $0.0000(-0.7)$ \\
\hline Log Price of vegetables at PSU level & $-0.842^{\star \star \star}(-2.69)$ & $-0.0002^{*}(-1.88)$ & $0.120(0.38)$ & $0.0000(0.37)$ \\
\hline Log Price of salt-spices at PSU level & $-0.388(-0.52)$ & $-0.0001(-0.50)$ & $0.534(0.79)$ & $0.0002(0.75)$ \\
\hline Log Price of tea-coffee at PSU level & $-0.388(-0.86)$ & $-0.0001(-0.81)$ & $-0.368(-0.83)$ & $-0.0001(-0.77)$ \\
\hline Log Price of paan-tobacco at PSU level & $-0.130 \star(-1.70)$ & $0.0000(-1.52)$ & $-0.294^{\star}(-1.89)$ & $-0.0001(-1.38)$ \\
\hline Log Price of fruits-nuts at PSU level & $0.478(1.10)$ & $0.0001(1.03)$ & $-0.139(-0.34)$ & $0.0000(-0.34)$ \\
\hline Constant & $13.531(1.27)$ & & $19.108^{\star \star}(2.20)$ & \\
\hline Number of observations & 57463 & & 37128 & \\
\hline LR chi-square & $75.15^{\star \star \star}$ & & $87.38^{\star \star \star}$ & \\
\hline Pseudo R-square & 0.1765 & & 0.2011 & \\
\hline Log likelihood & -175.308 & & -173.54128 & \\
\hline Predicted probability & & 0.0001 & & 0.0001 \\
\hline
\end{tabular}

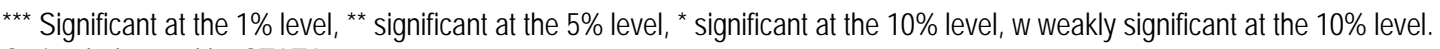
Omitted=dropped by STATA.

Let us now turn to the urban results. These results differ considerably from the rural results.

- The risk of cancer rises with age but at a diminishing rate. 
- The STs are less prone to it.

- The risk of cancer diminishes with expenditure but this relationship is weaker at higher expenditure levels.

- The price effects also differ: prices of wheat and cereal products are positively linked to this risk while those of sugar, and tea and coffee are negatively associated with it.

\section{Marginal Effects}

As all marginal effects are very small in both rural and urban samples, no further comment is necessary.

\section{Price, Expenditure and Nutrient Intake}

Now we analyse the effects of prices and expenditure on calories, proteins and fats. First, some comments are offered on the regression coefficients, followed by a discussion of the elasticities.

In the specification employed, nutrients (e.g. calories, proteins and fats per capita) are regressed on log of prices at the PSU level (village for rural areas and towns for urban areas) and (predicted) log of per capita expenditure and its square.

\section{(a) Calories}

We note from Table 37 that six price effects (e.g. wheat, other cereals, pulses, vegetables, salt and spices) on calorie intake are negative while seven are positive (e.g. rice, meat, gur, eggs, tea and coffee). The effect of expenditure on calorie intake is not significant but that of its square is positive and significant. The dummy for rural areas is significantly higher and that for urban metros significantly lower relative to non-metro urban areas.

The calorie-price elasticities are generally low. In absolute magnitude, the price elasticities are not-so small for fruits and nuts, pulses, tea and coffee, salt and spices, other cereals and gur. Expenditure elasticities are, however, negligible ${ }^{15}$.

\section{(b) Protein}

Price and expenditure effects on protein intake are given in Table 38. Six price effects (wheat, other cereals, pulses, milk, vegetables and fruits and nuts) are negative while seven (rice, meat, gur, salt and spices, tea and coffee, and pan and tobacco) are positive. The effect of expenditure is not significant while its square has a weakly significant positive effect.

Protein-price elasticities are moderately high for fruits and nuts, pulses, salt and spices, other cereals, and tea and coffee. Expenditure elasticities are not significant.

$$
\text { (c) Fats }
$$

Ten price effects (rice, cereal product, meat, gur, eggs, milk, vegetables, salt and spices, tea and coffee, and paan and tobacco) on fat intake are positive while 4 (other cereals, pulses, edible oil, and fruits and nuts) are negative. While per capita expenditure has a negative effect, its square has a positive effect, implying that the negative effect weakens at higher expenditure. While the rural dummy has a significant positive effect that of metros has a negative effect relative to the remaining urban areas.

\footnotetext{
${ }^{15}$ This is consistent with Behrman and Deolalikar (1990).
} 
Let us consider the price and expenditure elasticities in Table 39. Going by absolute magnitude, the price elasticities are high for tea and coffee, fruits and nuts, edible oil, milk and pulses. The expenditure elasticity is high too.

Table 37: Effect of Price and Expenditure on Per Capita Intake of Calorie in India: Robust Regression Estimates

\begin{tabular}{|c|c|c|}
\hline Explanatory variables & Coefficients(t-value) & Elasticity(z-value) \\
\hline Log Price of rice at PSU level & $-25.56(-1.10)$ & $-0.01(-1.1)$ \\
\hline Log Price of wheat at PSU level & $-65.73^{\star \star \star}(-2.69)$ & $-0.04^{\star \star \star}(-2.69)$ \\
\hline Log Price of sugar at PSU level & $6.66(0.13)$ & $0.00(0.13)$ \\
\hline Log Price of other cereals at PSU level & $-180.80^{\star \star \star}(-15.59)$ & $-0.10^{\star \star \star}(-15.58)$ \\
\hline Log Price of cereal product at PSU level & $14.89(0.74)$ & $0.01(0.74)$ \\
\hline Log Price ofr pulses at PSU level & $-329.54^{\star \star \star}(-14.15)$ & $-0.18^{\star \star \star(-14.15)}$ \\
\hline Log Price of meat at PSU level & $52.68^{\star \star \star}(3.44)$ & $0.03^{\star \star \star}(3.44)$ \\
\hline Log Price of gur at PSU level & $156.81^{\star \star \star(10.77)}$ & $0.08^{\star \star \star}(10.77)$ \\
\hline Log Price of edible-oil at PSU level & $27.98(0.53)$ & $0.02(0.53)$ \\
\hline Log Price of eggs at PSU level & $2.17(0.09)$ & $0.00(0.09)$ \\
\hline Log Price of milk at PSU level & $-32.39(-0.97)$ & $-0.02(-0.97)$ \\
\hline Log Price of milk-products at PSU level & $-39.55^{\star \star \star}(-5.30)$ & $-0.02^{\star \star \star}(-5.3)$ \\
\hline Log Price of vegetables at PSU level & $-82.11^{\star \star \star}(-4.69)$ & $-0.04^{\star \star *}(-4.69)$ \\
\hline Log Price of salt-spices at PSU level & $219.40^{\star \star \star}(5.89)$ & $0.12^{\star \star \star}(5.89)$ \\
\hline Log Price of tea-coffee at PSU level & $311.94^{\star \star \star(13.69)}$ & $0.17^{\star \star \star}(13.68)$ \\
\hline Log Price of paan-tobacco at PSU level & $25.25^{\star \star \star}(5.57)$ & $0.01^{\star \star \star}(5.57)$ \\
\hline Log Price of fruits-nuts at PSU level & $-732.31^{\star \star \star}(-32.48)$ & $-0.39 * \star *(-32.39)$ \\
\hline Log PCME (IV) & $-3.91(-0.02)$ & $0.00(-0.02)$ \\
\hline Square of Log PCME (IV) & $46.15^{\star \star \star}(2.58)$ & $0.02^{\star \star \star}(2.58)$ \\
\hline Rural & $181.22^{\star \star \star}(13.66)$ & $0.10 \star \star \star(13.66)$ \\
\hline Metro Urban & $-197.91^{\star \star \star}(-11.49)$ & $-0.11^{\star \star *}(-11.48)$ \\
\hline Constant & 1057.34(1.26) & \\
\hline Number of observations & 19005 & \\
\hline F-value & $201.15^{\star \star \star}$ & \\
\hline Predicted Calorie (mean) & & 1860.75 \\
\hline
\end{tabular}

Note: Breusch-Pagan / Cook-Weisberg test for heteroscedasticity in ordinary least squares regression with same functional form rejected the hypothesis of homoscedasticty at the $1 \%$ level of significance (chi-square(1)=1791.18). Therefore, robust regression is applied. ${ }^{* * *}$ Significant at the $1 \%$ level, ${ }^{* *}$ significant at the $5 \%$ level, ${ }^{*}$ significant at the $10 \%$ level

\section{Nutrients and Incidence of NCDs}

The focus here is on a health production function. A measure of average incidence of NCDs is defined in which number of household members suffering from either high blood pressure, heart disease, diabetes, or cancer is divided by household size. The right side variables include (predicted) intake of calories per capita, protein per capita, fats per capita, gender of household head, age and age squared of household head, highest educational attainments of adult males and females, log or per capita expenditure, caste affiliation (whether OBC, SC, or ST relative to Others). First, this analysis is carried out using a tobit model (since there is a large number of zeros and small positive values) on the complete sample, followed by separate analyses of rural and urban samples. Additional exercises using actual nutrient intakes are also carried out. 
Table 38: Effect of Price and Expenditure on Per Capita Intake of Protein in India: Robust Regression Estimates

\begin{tabular}{|c|c|c|}
\hline Explanatory variables & Coefficients(t-value) & Elasticity(z-value) \\
\hline Log of Price of rice at PSU level & $2.97^{\star \star \star}(4.56)$ & $0.06^{\star \star \star}(4.56)$ \\
\hline Log Price of wheat at PSU level & $-4.40^{\star \star \star}(-6.41)$ & $-0.09 * \star \star(-6.41)$ \\
\hline Log Price of sugar at PSU level & $-0.89(-0.63)$ & $-0.02(-0.63)$ \\
\hline Log Price of other cereals at PSU level & $-5.49 * \star *(-16.80)$ & $-0.11^{\star \star \star}(-16.79)$ \\
\hline Log Price of cereal product at PSU level & $0.51(0.89)$ & $0.01(0.89)$ \\
\hline Log Price of pulses at PSU level & $-16.65^{\star \star \star}(-25.40)$ & $-0.35^{\star \star \star}(-25.35)$ \\
\hline Log Price of meat at PSU level & $3.66^{\star \star \star}(8.48)$ & $0.08^{\star \star \star}(8.47)$ \\
\hline Log Price of gur at PSU level & $3.40^{\star \star \star}(8.30)$ & $0.07^{\star \star \star}(8.30)$ \\
\hline Log Price of edible-oil at PSU level & $2.02(1.35)$ & $0.04(1.35)$ \\
\hline Log Price of eggs at PSU level & $0.04(0.06)$ & $0.00(0.06)$ \\
\hline log Price of milk at PSU level & $-2.73^{\star \star \star}(-2.92)$ & $-0.06^{\star \star \star}(-2.92)$ \\
\hline Log Price of milk-products at PSU level & $-0.07(-0.35)$ & $0.00(-0.35)$ \\
\hline Log Price of vegetables at PSU level & $-1.84^{\star \star \star(-3.73)}$ & $-0.04^{\star \star \star}(-3.73)$ \\
\hline Food Price of salt-spices at PSU level & $16.00^{\star \star \star}(15.24)$ & $0.33^{\star \star \star}(15.23)$ \\
\hline Log Price of tea-coffee at PSU level & $5.36^{\star \star \star}(8.35)$ & $0.11^{\star \star \star}(8.35)$ \\
\hline Log Price of paan-tobacco at PSU level & $0.92^{\star \star \star}(7.20)$ & $0.02^{\star \star \star}(7.20)$ \\
\hline Log Price of fruits-nuts at PSU level & $-22.89 * \star *(-36.07)$ & $-0.48^{\star \star *}(-35.91)$ \\
\hline Log PCME (IV) & $4.52(0.68)$ & $0.09(0.68)$ \\
\hline Square of Log PCME (IV) & $0.74(1.47)$ & $0.02(1.47)$ \\
\hline Rural & $5.51^{\star \star \star}(14.76)$ & $0.12^{\star \star \star}(14.75)$ \\
\hline Metro Urban & $-5.51^{\star \star \star}(-11.36)$ & $-0.12^{\star \star \star}(-11.35)$ \\
\hline Constant & $7.83(0.33)$ & \\
\hline Number of observations & 19005 & \\
\hline F-value & $268.60^{\star \star \star}$ & \\
\hline Predicted protein (mean) & & $47.78(\mathrm{~g})$ \\
\hline
\end{tabular}

Note: Breusch-Pagan / Cook-Weisberg test for heteroscedasticity in ordinary least squares regression with same functional form rejected the hypothesis of homoscedasticty at $1 \%$ level of significance (chi-square $(1)=1676.83$ ). Therefore, robust regression is applied. *** Significant at the $1 \%$ level, ${ }^{* *}$ significant at the $5 \%$ level, ${ }^{*}$ significant at the $10 \%$ level

Let us first consider the rural results in Table 40. We will first comment on the tobit coefficients and then on the elasticities implied by them.

Calorie intake and incidence of NCDs are positively related while protein and fats are inversely related. Age of household head and incidence of NCDs are positively related but this relationship weakens with advancing age. Higher educational attainments of females reduce this incidence. Per capita expenditure is positively associated with it. Each of the three castes has a lower incidence relative to Others, further supporting the important of role of affluence in NCDs.

Going by absolute magnitude, the elasticity of incidence with respect to calories is highest, followed by that of protein. The elasticity with respect to age is high too except that it diminishes with advancing age. Finally, the expenditure elasticity is largest, confirming the strong association between NCDs and affluence. 
Table 39: Effect of Price and Expenditure on Per Capita Intake of Fats in India: Robust Regression Estimates

\begin{tabular}{|c|c|c|}
\hline Explanatory variables & Coefficients(t-value) & Elasticity(z-value) \\
\hline Log Price of rice at PSU level & $7.19^{\star \star \star}(9.76)$ & $0.20^{\star \star \star}(9.75)$ \\
\hline Log Price of wheat at PSU level & $0.09(0.12)$ & $0.00(0.12)$ \\
\hline Log Price of sugar at PSU level & $0.12(0.07)$ & $0.00(0.07)$ \\
\hline Log Price of other cereals at PSU level & $-5.00 * \star *(-13.57)$ & $-0.14^{\star \star \star}(-13.55)$ \\
\hline Log Price of cereal product at PSU level & $5.94 \star \star \star(9.31)$ & $0.16^{\star \star \star}(9.30)$ \\
\hline Log Price of pulses at PSU level & $-12.29 * * *(-16.61)$ & $-0.33^{\star \star \star}(-16.57)$ \\
\hline Log Price of meat at PSU level & $2.29 * \star(4.71)$ & $0.06^{\star \star \star}(4.71)$ \\
\hline Log Price of gur at PSU level & $3.39 \star \star \star(7.34)$ & $0.09 \star \star \star(7.33)$ \\
\hline Log Price of edible-oil at PSU level & $-19.97^{\star \star \star}(-11.83)$ & $-0.54^{\star \star \star}(-11.82)$ \\
\hline Log Price of eggs at PSU level & $2.71^{\star \star \star(3.36)}$ & $0.07^{\star \star \star}(3.36)$ \\
\hline Log Price of milk at PSU level & $12.80^{\star \star \star}(12.12)$ & $0.35^{\star \star \star}(12.11)$ \\
\hline Log Price of milk-products at PSU level & $0.07(0.28)$ & $0.00(0.28)$ \\
\hline Log Price of vegetables at PSU level & $2.77^{\star \star \star}(4.98)$ & $0.08^{\star \star \star}(4.98)$ \\
\hline Log Price of salt-spices at PSU level & $5.81^{\star \star \star}(4.91)$ & $0.16^{\star \star \star}(4.91)$ \\
\hline Log Price of tea-coffee at PSU level & $23.35^{\star \star \star}(32.25)$ & $0.63^{\star \star \star(32.01) ~}$ \\
\hline Log Price of paan-tobacco at PSU level & $0.35^{\star \star}(2.44)$ & $0.01 * *(2.44)$ \\
\hline Log Price of fruits-nuts at PSU level & $-20.66^{\star \star \star}(-28.84)$ & $-0.56^{\star \star \star}(-28.66)$ \\
\hline Log PCME (IV) & $-50.56^{\star \star \star}(-6.71)$ & $-1.37^{\star \star \star}(-6.71)$ \\
\hline Square of Log PCME (IV) & $5.53^{\star \star \star}(9.73)$ & $0.15^{\star \star \star}(9.73)$ \\
\hline Rural & $2.29 \star \star \star(5.42)$ & $0.06^{\star \star \star}(5.42)$ \\
\hline Metro Urban & $-12.77^{\star \star \star}(-23.32)$ & $-0.35^{\star \star \star}(-23.23)$ \\
\hline Constant & $110.91^{\star \star \star}(4.15)$ & \\
\hline Number of observations & 19005 & \\
\hline F-value & $471.04^{\star \star \star}$ & \\
\hline Predicted fats (mean) & & $36.85(\mathrm{~g})$ \\
\hline
\end{tabular}

Note: Breusch-Pagan / Cook-Weisberg test for heteroscedasticity in ordinary least squares regression with same functional form rejected the hypothesis of homoscedasticty at the $1 \%$ level of significance (chi-square(1)==3711.21).

Therefore, robust regression is applied. ${ }^{* \star *}$ Significant at the $1 \%$ level, ** significant at the $5 \%$ level, * significant at

The results for the urban sample are given in Table 41. Many of the effects are similar. Calorie intake is positively linked to incidence of NCDs while protein and fats are negatively associated with it. Age enhances this incidence as also female education. Moreover, the higher the per capita expenditure, the higher is the incidence. OBCs, SCs and

STs-especially the last two-have significantly lower incidence than Others, further corroborating the important role of affluence as a causal factor.

The elasticities help assess relative importance of these associations. Both calories and protein have high elasticities, as also age. Expenditure, however, has the highest elasticity. It is in fact much larger than that obtained from the rural sample. 
Table 40: Factors Associated with Average Incidences of NCDs in Rural India: Tobit analysis (with predicted nutrient intakes)

\begin{tabular}{|c|c|c|}
\hline Explanatory variables & Coefficient(t-value) & ey/ex (z-value) \\
\hline Per capita calorie intake (predicted) & $0.0005^{\star \star \star}(4.87)$ & $1.2821^{\star \star \star}(4.89)$ \\
\hline Per capita protein intake (predicted) & $-0.0142^{\star \star \star}(-5.04)$ & $-0.9541^{\star \star \star}(-5.07)$ \\
\hline Per capita fat intake (predicted) & $-0.0064^{\star \star \star}(-7.12)$ & $-0.2962^{\star \star \star}(-7.16)$ \\
\hline Gender & $-0.0458(-1.33)$ & $-0.0027(-1.33)$ \\
\hline Age & $0.0093^{\star \star \star}(2.72)$ & $0.6512^{\star \star \star}(2.73)$ \\
\hline Square of age & $-0.0001 * *(-2.24)$ & $-0.2710^{* *}(-2.24)$ \\
\hline Highest education: Female & $0.0072^{\star \star \star}(3.81)$ & $0.0405^{\star \star \star}(3.84)$ \\
\hline Highest education: Male & $-0.0004(-0.22)$ & $-0.0040(-0.22)$ \\
\hline Log PCME (IV) & $0.2516^{\star \star \star}(7.18)$ & $2.2318^{\star \star \star}(7.10)$ \\
\hline $\mathrm{OBC}$ & $-0.0360 *(-1.70)$ & $-0.0100 *(-1.70)$ \\
\hline SC & $-0.1117^{\star \star \star}(-2.67)$ & $-0.0091 * \star \star(-2.68)$ \\
\hline ST & $-0.0327^{\star *}(-1.98)$ & $-0.0188 \star *(-1.98)$ \\
\hline Constant & $-2.0624^{\star \star \star}(-9.67)$ & \\
\hline Sigma & 0.3032 & \\
\hline Number of observations & 3243 & \\
\hline LR Chi-square value & $508.28^{\star \star *}$ & \\
\hline Pseudo R-square & 0.1466 & \\
\hline Log likelihood & -1479.4397 & \\
\hline Expected value of $y$ conditional on $0<y<1$ & & 0.1958 \\
\hline
\end{tabular}

*** Significant at the $1 \%$ level, ${ }^{*}$ significant at the $5 \%$ level, * significant at the $10 \%$ level, w weakly significant at the $10 \%$ level. ey/ex=elasticity

The all-India results are given in Table 42. As in the rural and urban sample, many of the results are similar. Hence our comments are confined to the elasticities. The elasticities reveal a pattern not dissimilar from that observed in the rural and urban samples. Expenditure has the largest elasticity, followed by that of calories and proteins, and then education.

We note from Table 43 that a different measure of incidence of NCDs-in which a person reported more than one disease are added up and divided by household size- varies positively with calorie intake and negatively with protein and fat intakes. Households headed by women show lower incidence relative to the male-headed ones. There is a positive relationship between household head's age but this relationship weakens among older heads. The higher the (predicted) expenditure per capita the higher is the incidence, implying its association with affluence. This finding is further reinforced by the fact that all caste groups (OBCs, SCs and STs) have lower incidence of these diseases than Others (who are generally better-off). Locationally, rural areas which are generally worst-off in terms of living standards also display a lower incidence than non-metro urban areas.

Among the nutrients, large elasticities are associated with calories and protein, and it is highest with expenditure. 
The same model was run with actual nutrient intakes and all other variables as in Table $43 .{ }^{16}$. As many results are similar, our comments are confined to the elasticities. All nutrient and expenditure elasticities are generally lower with actual intake.

Table 41: Factors Associated with Average Incidences of NCDs in Urban India: Tobit analysis (with predicted nutrient intakes)

\begin{tabular}{|c|c|c|}
\hline Explanatory variables & Coefficient(t-value) & ey/ex (z-value) \\
\hline Per capita calorie intake (predicted) & $0.0003^{\star \star \star}(4.45)$ & $1.1856 \star \star \star(4.44)$ \\
\hline Per capita protein intake (predicted) & $-0.0132^{\star \star *}(-5.75)$ & $-1.1368^{\star \star \star}(-5.76)$ \\
\hline Per capita fat intake (predicted) & $-0.0019 * \star(-2.04)$ & $-0.1465^{\star \star}(-2.04)$ \\
\hline Gender & $-0.0129(-0.51)$ & $-0.0014(-0.51)$ \\
\hline Age & $0.0079 \star \star \star(2.64)$ & $0.7591 * \star \star(2.65)$ \\
\hline Square of age & $0.0000(-0.86)$ & $-0.1236(-0.86)$ \\
\hline Highest education: Female & $-0.0041^{\star * *}(-2.78)$ & $-0.0592^{\star * *}(-2.77)$ \\
\hline Highest education: Male & $-0.0012(-0.68)$ & $-0.0229(-0.68)$ \\
\hline Log PCME (IV) & $0.2511^{\star * \star}(7.36)$ & $3.3354^{\star \star \star}(7.25)$ \\
\hline $\mathrm{OBC}$ & $-0.0276(-1.53)$ & $-0.0080(-1.53)$ \\
\hline $\mathrm{SC}$ & $-0.1639 * \star *(-2.61)$ & $-0.0040^{\star \star *}(-2.61)$ \\
\hline ST & $-0.0439 * * *(-3.23)$ & $-0.0302^{\star \star *}(-3.23)$ \\
\hline Metro Urban & $-0.0130(-0.73)$ & $-0.0063(-0.73)$ \\
\hline Constant & $-1.8884^{\star \star \star}(-9.47)$ & \\
\hline Sigma & 0.2433 & \\
\hline Number of observations & 2120 & \\
\hline LR Chi-square value & $493.33^{\star \star *}$ & \\
\hline Pseudo R-square & 0.2456 & \\
\hline Log likelihood & -757.62927 & \\
\hline Expected value of $y$ conditional on $0<y<1$ & 0.2157 & \\
\hline
\end{tabular}

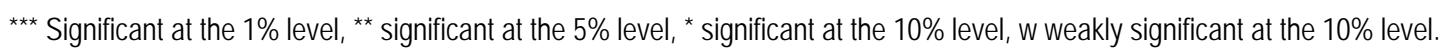
ey/ex=elasticity

\section{Concluding Observations}

Growing affluence of Indians, dietary transition and higher prevalence rates of NCDs especially diabetes-set the stage for the preceding analysis. As these diseases are associated with enormous economic burden on households and macro effects through lower savings, lower labour productivity and growth decelerations, it is necessary to understand the underlying factors. Towards this objective, a detailed econometric analysis was carried out using different specifications of health outcomes. In one set of specifications, incidence of each of the four major NCDs-high blood pressure, heart disease, diabetes and cancer- is analysed in which individual, household and village characteristics are used as explanatory variables. A central role was assigned to various food prices at the village or town level (PSUs). The analysis confirmed significant price effects working through changes in consumption patterns and associated with them changes in nutrient intake. Although these prices show robust associations with various diseases, their marginal effects are generally small. Somewhat surprisingly, the effects of per capita expenditure are also small.

\footnotetext{
${ }^{16}$ Details will be furnished on request.
} 
Table 42: Factors associated with Average incidences of NCDs in All India: Tobit analysis (with predicted nutrient intakes)

\begin{tabular}{|c|c|c|}
\hline Explanatory variables & Coefficient(t-value) & ey/ex (z-value) \\
\hline Per capita calorie intake (predicted) & $0.0004^{* \star *}(7.21)$ & $1.3348^{\star \star \star}(7.22)$ \\
\hline Per capita protein intake (predicted) & $-0.0139 * \star \star(-7.93)$ & $-1.0597 * \star(-7.95)$ \\
\hline Per capita fat intake (predicted) & $-0.0045^{\star \star \star}(-7.17)$ & -0.2601 ***(-7.17) \\
\hline Gender & $-0.0239(-1.14)$ & $-0.0018(-1.14)$ \\
\hline Age & $0.0094^{\star \star *}(4.15)$ & $0.7642^{\star \star \star}(4.15)$ \\
\hline Square of age & $-0.0001^{* * *(-2.71)}$ & $-0.2514^{\star \star \star}(-2.71)$ \\
\hline Rural & $-0.0554^{\star \star \star}(-4.52)$ & $-0.0575^{\star \star \star}(-4.53)$ \\
\hline Metro Urban & $-0.0087(-0.53)$ & $-0.0014(-0.53)$ \\
\hline Highest education: Female & $0.0016(1.35)$ & $0.0137(1.36)$ \\
\hline Highest education: Male & $0.0002(0.15)$ & $0.0024(0.15)$ \\
\hline Log PCME (IV) & $0.2334^{\star \star \star}(10.07)$ & $2.4828^{\star \star \star}(9.96)$ \\
\hline $\mathrm{OBC}$ & $-0.0312^{\star \star}(-2.28)$ & $-0.0092 * *(-2.28)$ \\
\hline $\mathrm{SC}$ & $-0.1084^{\star \star \star}(-3.45)$ & $-0.0074^{\star \star \star}(-3.46)$ \\
\hline ST & $-0.0351^{\star \star *}(-3.33)$ & $-0.0224^{\star \star *}(-3.34)$ \\
\hline Constant & $-1.8774^{\star \star \star}(-13.39)$ & \\
\hline Sigma & 0.2725 & \\
\hline Number of observations & 5363 & \\
\hline LR Chi-square value & $1127.41^{\star \star \star}$ & \\
\hline Pseudo R-square & 0.1964 & \\
\hline Log likelihood & -2307.11 & \\
\hline Expected value of y conditional on $0<y<1$ & & 0.1970 \\
\hline
\end{tabular}

${ }^{*}{ }^{* \star}$ Significant at the $1 \%$ level, ${ }^{*}$ significant at the $5 \%$ level, ${ }^{*}$ significant at the $10 \%$ level, w weakly significant at the $10 \%$ level. ey/ex=elasticity

To understand better how these price changes induce substitutions between commodities with significant nutritional effects, we analysed the changes in calories, protein and fats. Predicted values of these nutrients and various household characteristics are then cast as explanatory variable in a health production function with two alternative measure of average incidence of NCDs per household. In one measure, our average refers to number of persons reporting suffering from any of the four NCDs divided by household size. In another, taking account of the fact that there are overlaps between various diseases (e.g. between high blood pressure and heart disease, between diabetes and heart disease) and some individuals report suffering from more than one ailment, we added up the different ailments reported by household members divided by household size. Robust effects of nutrients (e.g. calories enhance the incidence of NCDs), expenditure and caste affiliations were obtained. In general, the important roles of dietary patterns and (relative) affluence are corroborated.

In conclusion, these results raise serious concerns about consumption patterns that have evolved in a period of prosperity. While our analysis points to the potential role of price subsidies, the complexity of nutritional outcomes and associated risk of NCDs need careful scrutiny. 
Table 43: Factors Associated with Number of Ailments per person in India: Tobit analysis (with predicted nutrient intakes)

\begin{tabular}{|c|c|c|}
\hline Explanatory variables & Coefficient(t-value) & ey/ex (z-value) \\
\hline Per capita calorie intake (predicted) & $0.0005^{\star \star \star}(6.25)$ & $1.1586^{\star \star \star}(6.26)$ \\
\hline Per capita protein intake (predicted) & $-0.0174^{\star \star \star}(-7.59)$ & $-1.0169 * \star \star(-7.61)$ \\
\hline Per capita fat intake (predicted) & $-0.0057^{\star * *}(-6.96)$ & $-0.2531^{\star \star \star}(-6.96)$ \\
\hline Gender & $-0.0467^{\star}(-1.71)$ & $-0.0027^{\star}(-1.71)$ \\
\hline Age & $0.0118^{\star \star \star}(3.99)$ & $0.7379^{\star \star \star}(4.00)$ \\
\hline Square of age & $-0.0001^{\star *}(-2.48)$ & $-0.2311 * *(-2.49)$ \\
\hline Rural & $-0.0535^{\star \star \star}(-3.37)$ & $-0.0428^{\star \star \star}(-3.37)$ \\
\hline Metro Urban & $-0.0157(-0.73)$ & $-0.0019(-0.73)$ \\
\hline Highest education: Female & $0.0025^{\star}(1.63)$ & $0.0165^{\star}(1.63)$ \\
\hline Highest education: Male & $0.0004(0.24)$ & $0.0039(0.24)$ \\
\hline Log PCME (IV) & $0.3244^{\star \star \star}(10.74)$ & $2.6549^{\star \star \star}(10.63)$ \\
\hline $\mathrm{OBC}$ & $-0.0469 * \star *(-2.62)$ & $-0.0107^{\star \star \star}(-2.62)$ \\
\hline $\mathrm{SC}$ & $-0.1471^{* * *(-3.58)}$ & $-0.0077^{\star \star \star}(-3.59)$ \\
\hline ST & $-0.0463^{\star \star \star}(-3.38)$ & $-0.0227^{\star \star \star}(-3.38)$ \\
\hline Constant & $-2.5019 * \star *(-13.70)$ & \\
\hline Sigma & 0.3523 & \\
\hline Number of observations & 5363 & \\
\hline LR Chi-square value & $1173.49 * * *$ & \\
\hline Pseudo R-square & 0.1726 & \\
\hline Log likelihood & -2813.2222 & \\
\hline Expected value of $y$ conditional on $0<y<1$ & & 0.2492 \\
\hline
\end{tabular}

*** Significant at the $1 \%$ level, ${ }^{* *}$ significant at the $5 \%$ level, * significant at the $10 \%$ level, w weakly significant at the $10 \%$ level. ey/ex=elasticity

\section{References}

Bajpai, N., J. Sachs and R.H. Dholakia (2010) Improving Access and Efficiency of in Public health Services: Mid-Term Evaluation of India's National Rural Health Mission, New Delhi: Sage.

Behrman, J. and A. Deolalikar (1990) 'The Intrahousehold Demand for Nutrients in Rural South India: Individual Estimates, Fixed Effects and Permanent Income’, The Journal of Human Resources, 25(4).

Bloom, D., D. Canning and G. Fink (2009) 'Disease and Development Revisited', Cambridge: MA : NBER Working Paper 15137.

Chaudhury, N., J.S. Hammer and A. Pokharel (2008) 'First Things First: Infectious Disease, Child Mortality and the Poor in India 1992-2005’, Washington DC: World Bank, mimeo.

Deasai, S., A. Dubey, B. Joshi, M. Sen, A. Shariff, and R. Vanneman (2010) Human Development in India: Challenges for a Society in Transition, New Delhi: Oxford University Press.

Mahal, A., A. Karan and M. Engelan (2009) The Economic Implications of Non-Communicable Disease for India, Washington DC: World Bank, mimeo.

Popkin, B., S. Horton, S. Kim, A. Mahal, and J. Shuigao (2001) 'Trends in Diet, Nutritional Status, and DietRelated Non-Communicable Diseases in China and India: The Economic Costs of the Nutrition Transition', Nutrition Reviews, 59.

Wooldridge, J. M. (2006) Introductory Econometrics: A Modern Approach, Third Edition, Thomson South Western. 
Table A.1 Definitions of Variable Used

\begin{tabular}{|c|c|}
\hline Variables & Definition \\
\hline \multicolumn{2}{|r|}{ Dependent variables } \\
\hline High Blood Pressure & $=1$ if person suffers from high blood pressure; 0 otherwise \\
\hline Heart Disease & $=1$ if person suffers from heart disease; 0 otherwise \\
\hline Diabetes & $=1$ if person suffers from diabetes; 0 otherwise \\
\hline Cancer & $=1$ if person suffers from cancer; 0 otherwise \\
\hline Per capita calorie intake & $=$ Total per person per day calorie intake (in Kcal) \\
\hline Per capita protein intake & $=$ Total per person per day protein intake (in gram) \\
\hline Per capita fat intake & $=$ Total per person per day fat intake (in gram) \\
\hline Average incidence of NCDs & $\begin{array}{c}=\text { Number of household members suffering from either high blood pressure, } \\
\text { heart disease, diabetes, or cancer divided by household size (range: } 0 \text { to } 1 \text { ) }\end{array}$ \\
\hline Number of ailments per person & =Number of ailments reported by all household members/household size \\
\hline \multicolumn{2}{|r|}{ Explanatory variables } \\
\hline Age & Age (years) \\
\hline Square of age & Square of age \\
\hline Gender & $=1$ if female; 0 if male \\
\hline Years of education & Number of years completed in education \\
\hline Square of years of education & Square of years of education \\
\hline Highest education: Female & $\begin{array}{l}\text { Number of years completed in education by most educated female in the } \\
\text { household }\end{array}$ \\
\hline Highest education: Male & $\begin{array}{l}\text { Number of years completed in education by most educated male in the } \\
\text { household }\end{array}$ \\
\hline Other (reference) & $=1$ if social group is other; 0 otherwise \\
\hline SC & $=1$ if social group is $\mathrm{SC} ; 0$ otherwise \\
\hline ST & $=1$ if social group is ST; 0 otherwise \\
\hline $\mathrm{OBC}$ & $=1$ if social group is $\mathrm{OBC} ; 0$ otherwise \\
\hline Log PCME (IV) & Log of per capita monthly expenditure (predicted-) \\
\hline Square of Log PCME (IV) & Square of ( predicted) log of per capita monthly expenditure \\
\hline Village distance from nearest hospital & Distance of nearest hospital from the village (in km) \\
\hline Metro & $=1$ if urban area is a metro; 0 otherwise \\
\hline Slum & $=1$ if urban area is a slum; 0 otherwise \\
\hline Log Price of rice & Log of food price of rice at PSU level (in Rs.) \\
\hline Log Price of wheat & Log of food price of wheat at PSU level (in Rs.) \\
\hline Log Price of sugar & Log of food price of sugar at PSU level (in Rs.) \\
\hline Log Price of other cereals & Log of food price of other cereals at PSU level \\
\hline Log Price of cereal product & Log of food price of cereal product at PSU level (in Rs.) \\
\hline Log Price of pulses & Log of food price of pulses at PSU level (in Rs.) \\
\hline Log Price of meat & Log of food price of meat at PSU level (in Rs.) \\
\hline Log Price of gur & Log of food price of gur at PSU level (in Rs.) \\
\hline Log Price of edible-oil & Log of food price of edible-oil at PSU level (in Rs.) \\
\hline Log Price of eggs & Log of food price of eggs at PSU level (in Rs.) \\
\hline Log Price of milk & Log of food price of milk at PSU level (in Rs.) \\
\hline Log Price of milk-products & Log of food price of milk-products at PSU level (in Rs.) \\
\hline Log Price of vegetables & Log of food price of vegetables at PSU level (in Rs.) \\
\hline Log Price of salt-spices & Log of food price of salt-spices at PSU level (in Rs.) \\
\hline Log Price of tea-coffee & Log of food price of tea-coffee at PSU level (in Rs.) \\
\hline Log Price of paan-tobacco & Log of food price of paan-tobacco at PSU level (in Rs.) \\
\hline Log Price of fruits-nuts & Log of food price of fruits-nuts at PSU level (in Rs.) \\
\hline
\end{tabular}

Note: For rural areas, PSU is a village, and, for urban areas, it is a town. 
Table A.2

Factors Associated with Heart Disease in India: Probit Analysis (with height)

\begin{tabular}{|c|c|c|}
\hline Explanatory variables & Coeff(t-value) & Marginal effect(z-value) \\
\hline Age & $0.0392^{\star \star \star}(2.78)$ & $0.0002^{\star \star \star}(2.90)$ \\
\hline Square of age & $-0.0001(-0.65)$ & $0.0000(-0.66)$ \\
\hline Gender & $-0.1085(-0.85)$ & $-0.0006(-0.79)$ \\
\hline Years of education & $0.0629 * *(2.51)$ & $0.0003^{\star \star}(2.43)$ \\
\hline Square of years of education & $-0.0048^{\star \star}(-2.28)$ & $-0.00003^{\star \star}(-2.22)$ \\
\hline Height & $-0.0064^{\star *}(-2.33)$ & $-0.00003^{\star \star}(-2.31)$ \\
\hline $\mathrm{OBC}$ & $-0.1347(-0.58)$ & $-0.0006(-0.69)$ \\
\hline SC & $0.0782(0.77)$ & $0.0004(0.75)$ \\
\hline ST & $0.1570(1.51)$ & $0.0009(1.39)$ \\
\hline Log PCME (IV) & $1.5782(0.74)$ & $0.0085(0.74)$ \\
\hline Square of Log PCME (IV) & $-0.1148(-0.71)$ & $-0.0006(-0.71)$ \\
\hline Rural & $-0.0684(-0.66)$ & $-0.0004(-0.64)$ \\
\hline Metro & $-0.4380^{\star \star}(-2.54)$ & $-0.0015^{\star \star}(-3.94)$ \\
\hline Log Price of rice & $-0.1665(-0.86)$ & $-0.0009(-0.86)$ \\
\hline Log Price of wheat & $0.1698(0.83)$ & $0.0009(0.83)$ \\
\hline Log Price of sugar & $-0.6726^{\star}(-1.67)$ & $-0.0036 *(-1.67)$ \\
\hline Log Price of other cereals & $-0.1151(-1.34)$ & $-0.0006(-1.33)$ \\
\hline Log Price of cereal product & $-0.2403(-1.55)$ & $-0.0013(-1.53)$ \\
\hline Log Price of pulses & $-0.2930(-1.53)$ & $-0.0016(-1.52)$ \\
\hline Log Price of meat & $-0.1281(-1.04)$ & $-0.0007(-1.04)$ \\
\hline Log Price of gur & $0.0167(0.16)$ & $0.0001(0.16)$ \\
\hline Log Price of edible-oil & $0.8093^{\star}(1.89)$ & $0.0044^{*}(1.88)$ \\
\hline Log Price of eggs & $-0.3968 *(-1.80)$ & $-0.0021 *(-1.80)$ \\
\hline Log Price of milk & $0.1091(0.44)$ & $0.0006(0.44)$ \\
\hline Log Price of milk-products & $0.0215(0.43)$ & $0.0001(0.43)$ \\
\hline Log Price of vegetables & $-0.1045(-0.79)$ & $-0.0006(-0.79)$ \\
\hline Log Price of salt-spices & $0.3045(0.97)$ & $0.0016(0.97)$ \\
\hline Log Price of tea-coffee & $0.0938(0.47)$ & $0.0005(0.47)$ \\
\hline Log Price of paan-tobacco & $0.0286(0.76)$ & $0.0002(0.76)$ \\
\hline Log Price of fruits-nuts & $-0.4079 \star *(-2.42)$ & $-0.0022^{\star \star}(-2.38)$ \\
\hline Constant & $-6.1999(-0.83)$ & \\
\hline Number of observations & 32309 & \\
\hline LR chi-square & $172.28^{\star \star \star}$ & \\
\hline Pseudo R-square & 0.1130 & \\
\hline Log likelihood & -676.31791 & \\
\hline Predicted probability & & 0.0017 \\
\hline
\end{tabular}

${ }^{* \star \star}$ Significant at the $1 \%$ level, ${ }^{* \star}$ significant at the $5 \%$ level, * significant at the $10 \%$ level, w weakly significant at the $10 \%$ level. 